\title{
Mechanism of Earthquake Simulation as a Prenatal Stressor Retarding Rat Offspring Development and Chinese Medicine Correcting the Retardation: Hormones and Gene-Expression Alteration
}

\author{
X. G. Zhang, ${ }^{1}$ H. Zhang, ${ }^{1}$ R. Tan, ${ }^{2}$ J. C. Peng, ${ }^{3}$ X. L. Liang, ${ }^{1}$ Q. Liu, ${ }^{1}$ M. Q. Wang, ${ }^{4}$ and X. P. Yu ${ }^{5}$ \\ ${ }^{1}$ The School of Nursing, Chengdu University of T.C.M., Chengdu 610075, China \\ ${ }^{2}$ School of Biology and Engineering, Southwest Jiaotong University, Chengdu 610031, China \\ ${ }^{3}$ Earthquake Emergency Security Center, Sichuan Provincial Seismological Bureau, Chengdu 610041, China \\ ${ }^{4}$ Molecular Laboratory of T.C.M., Chengdu University of T.C.M., Chengdu 610075, China \\ ${ }^{5}$ School of Humanity and Information Management, Chengdu Medical University, Chengdu 610075, China \\ Correspondence should be addressed to X. P. Yu, 18908006961@189.cn
}

Received 26 August 2012; Revised 3 October 2012; Accepted 5 October 2012

Academic Editor: Jang-Hern Lee

Copyright (๑) 2012 X. G. Zhang et al. This is an open access article distributed under the Creative Commons Attribution License, which permits unrestricted use, distribution, and reproduction in any medium, provided the original work is properly cited.

We aimed to investigate the mechanism of shaking as a prenatal stressor impacting the development of the offspring and Chinese medicines correcting the alterations. Pregnant rats were randomized into earthquake simulation group (ESG), herbal group (HG) which received herbal supplements in feed after shaking, and control group (CG). Findings revealed body weight and open field test (OFT) score of ESG offspring were statistically inferior to the CG and HG offspring. The corticosterone levels of ESG were higher than those of CG but not than HG. The dopamine level of ESG was slightly lower than that of the CG and of HG was higher than that of ESG. The 5-HT of ESG was higher than CG and HG. The growth hormone level of the ESG was significantly lower than ESG but not than CG. Gene expression profile showed 81 genes upregulated and 39 genes downregulated in ESG versus CG, and 60 genes upregulated and 28 genes downregulated in ESG versus HG. Eighty-four genes were found differentially expressed in ESG versus CG comparison and were normalized in ESG versus HG. We conclude that maternal shaking negatively affected physical and nervous system development, with specific alterations in neurohormones and gene expression. Chinese herbal medicine reduced these negative outcomes.

\section{Introduction}

Maternal effects have been demonstrated as an essential factor for offspring development in many species. Because of the long period of perinatal mother-infant interaction in mammals, the growth and development and variations of offspring are very likely to be influenced by maternal impacts, leaving long-term consequences for both psychological and physiological health [1]. Recent human studies have shown that long-lasting and a wide variety of prenatal stressors, from anxiety and partner relationship problems to natural disasters, increase the risk for a diverse range of adverse neurodevelopmental outcomes in the child, including impaired cognitive development and behavioral problems [2, 3]. Animal experiments have convincingly demonstrated that prenatal maternal stress affects pregnancy outcome and results in early programming of brain functions with permanent changes in neuroendocrine regulation, gene expression, and behavior in offspring [4]. Prenatal restraint stress in rats is a common experimental model of early stress known to have long-term behavioral and neurobiological consequences [5, 6]. PS modifies the plastic responses of the adult brain, including the circuitry of the hippocampus-hypothalamuspituitary-adrenalaxis (HHPA), that participate in the neuroendocrine control of feeding and metabolism in adult life [7]. 
As a typical prenatal stress, shaking can significantly impact the psychological and intellectual development of fetus and birth outcomes [8] in human. Naturally, earthquake is a fierce shaking. Tan et al. [9] reported that rates of birth defects after an earthquake were significantly higher than those before earthquake, whose spectrum was dramatically altered after earthquake, with the markedly increased occurrences of ear malformations; meanwhile the ratio of preterm birth after earthquake was significant increased than that of before earthquake. Oyarzo et al. [10] reported that women exposed to the February 27th 2010 Chilean earthquake during her first trimester delivered smaller newborns and they were more likely diagnosed with early preterm delivery, preterm delivery, and PROM but were less likely diagnosed with intrauterine growth retardation and late delivery compared to those exposed at third trimester, indicating disasters such as earthquakes are associated to adverse perinatal outcomes that impact negatively the entire maternal-neonatal healthcare system. Like the other alterations induced by PS in behavior those in learning and their direction appears to be dependent on the intensity, duration, and timing of the maternal stress [11].

In Chinese medicine, PS from shaking or an analog of earthquake is considered as a factor which impairs kidney Qi (shen qi) [12]. As kidney is the root of earlier heaven (the congenital constitution), it governs reproduction and development and holds oriffice of labor, whence agility and emanates. Jin Kui Shen Qi Wan (JKSQW) is a typical herbal formula supplementing kidney Qi, which recovers the physiological functions of kidney [13].

The current study involves shaking as a prenatal stressor. A first goal was to establish that earthquake simulation led to significant delays in development. A second goal was to examine whether Chinese traditional medicine could be used to address these negative effects. Based on the above information, we hypothesized parental kidney is injured from PS derived from earthquake simulation on rats, traits are handed down to offspring, showing development retardation; JKSQW could recover the dysfunctions of kidney whose underlying mechanism could involve development, hormones and gene expression alterations.

\section{Materials and Methods}

2.1. Grouping. Forty-five Sprague-Dawley (SD) female rats $(230 \mathrm{~g} \sim 270 \mathrm{~g})$ and 45 male rats $(225 \mathrm{~g} \sim 261 \mathrm{~g})$ were involved in this research. The rats were housed in a room with a temperature of $22^{\circ} \mathrm{C}, 12$ hour light/dark cycle and fed with food and water ad libitum. After a week of adaption housing, the female rats were mated with the male rats. Pregnancy was confirmed by vaginal plug test. Then the 34 pregnant rats were randomized into three groups, control group (CG) $(n=11)$, earthquake simulation with conventional chow group (ESG) $(n=11)$, and earthquake plus herbal group (HG) $(n=12)$, and they were housed under pregnant rat cages until the delivery. With this procedure, all the groups were transferred with equivalent stress during pregnancy. There was no statistical difference of gestation time detected or body weight of the first day of gestation (CG: 234.87 \pm 2.20 , ESG: $234.98 \pm 1.95$, and HG: $235.16 \pm 1.96$, ANOVA test, $P>0.05(\mathrm{~g}))$ in the three groups. After delivery, all the litters of the three groups were housed with their mothers until the 25th day after birth.

2.2. Earthquake Simulation. The ESG cages housing pregnant rats were manually shaken up and down 3 times to simulate an initial earthquake and then were shaken for 50 timesover the next 15 minutes to modulate an aftershock [14]. The shaking was performed twice a day until delivery. Severity of the shake was measured with a seism velometer (DX-6Y2, Cheng Du Mei Huan Tech. Co. Ltd.), showing 9.6 10.5 of seismic intensity, $950 \mathrm{mg} \sim 1050 \mathrm{mg}$ of vertical peak ground accelerations (PGA), which was similar to the PGA (1080 mg) of Wenchuan earthquake, May 12, 2008, China.

2.3. Chinese Herbal Formula Feed. The feed of HG rats was supplemented with herbal medicine until delivery, which consisted of (Radix Rehmanniae Preparata (Shu Di Huang), Fructus Corni Officinalis (Shan Zhu Yu), Cortex Moutan Radicis (Mu Dan Pi), Rhizoma Dioscoreae Oppositae (Shan Yao), Sclerotium Poriae Cocos (Fu Ling), Rhizoma Alismatis Orientalis (Ze Xie), Radix Aconiti Lateralis Preparata (Zhi Fu $\mathrm{Zi}$ ), and Cortex Cinnamomi Cassiae (Rou Gui)) bought from Tong Ren Tang Technologies, Co., Ltd. The pill of JKSQW was grinded and added to the conventional feed $0.5 \sim 0.6 \mathrm{~g} / \mathrm{d}$.

2.4. Body Weight Measurement. Body weight (g) was measured at the 1 st (day 0$), 5$ th (day 5$), 10$ th (day 10), 15th (day 15), 20th (day 20), and 25th (day 25) days after delivery in order to evaluate the body development of the offspring.

2.5. Open Field Test (OFT). A square board $(90 \mathrm{~cm} \times 90 \mathrm{~cm})$ painted with yellow and white squares $(15 \mathrm{~cm} \times 15 \mathrm{~cm})$. The offspring of 25 days old was placed in the center of the board. We counted how many squares the offspring had crawled across in two minutes. One score was given only when the four paws of an offspring were in one square.

2.6. Hormone Assay. Thirty offspring were randomly selected from the groups, ten for each. Blood sample was taken from arteria femoralis. ELISA (R\&D Systems China Co., Ltd.) was employed to determine the serum level of corticosterone (DZE 30590), dopamine (DZE 30238), 5-HT (DZE 30326), and growth hormone (DZE 30549).

\subsection{Gene Expression Profile Chip Experiments}

2.7.1. RNA Extraction and Purification. Total RNA was extracted using TRIZOL Reagent (Cat no. 15596-018, technologies, Carlsbad, CA, US) following the manufacturer's instructions and checked for a RIN number to inspect RNA integration by an Agilent Bioanalyzer 2100 (Agilent technologies, Santa Clara, CA, US). Qualified total RNA was further purified by RNeasy mini kit (Cat no. 74106, QIAGEN, GmBH, Germany) and RNeasy micro kit (Cat no. 
TABLE 1: QC of RNA extraction and slides experiment (A sample is qualified only when $2100 \mathrm{RIN} \geq 7.0$ and $28 \mathrm{~S} / 18 \mathrm{~S} \geq 0.7$ ).

\begin{tabular}{|c|c|c|c|c|c|c|c|c|c|}
\hline \multirow{3}{*}{ Group } & \multicolumn{6}{|c|}{ QC of RNA } & \multirow{3}{*}{ Result } & \multicolumn{2}{|c|}{ QC of slides } \\
\hline & \multirow{2}{*}{ Con. $(\mu \mathrm{g} / \mu \mathrm{L})$} & \multirow{2}{*}{ Vol. $(\mu \mathrm{L})$} & \multirow{2}{*}{ Total $(\mu \mathrm{g})$} & \multirow{2}{*}{ A260/A280 } & \multicolumn{2}{|c|}{2100 Result } & & \multirow{2}{*}{$\mathrm{CV}(\%)^{*}$} & \multirow{2}{*}{ Detection rate $(\%)$} \\
\hline & & & & & RIN & $28 \mathrm{~S} / 18 \mathrm{~S}$ & & & \\
\hline \multirow{3}{*}{ ESG } & 0.168 & 50 & 8.41 & 1.88 & 9.4 & 1.7 & Qualified & 3.91 & 69.50 \\
\hline & 1.366 & 30 & 40.98 & 1.93 & 9.5 & 1.8 & Qualified & 4.85 & 62.96 \\
\hline & 0.246 & 50 & 12.29 & 1.90 & 9.4 & 1.7 & Qualified & 6.70 & 72.13 \\
\hline \multirow{3}{*}{ HG } & 0.134 & 50 & 6.69 & 1.81 & 9.4 & 1.8 & Qualified & 4.76 & 70.40 \\
\hline & 0.138 & 50 & 6.92 & 1.82 & 9.4 & 1.8 & Qualified & 4.90 & 72.30 \\
\hline & 0.372 & 50 & 18.58 & 1.86 & 9.5 & 1.6 & Qualified & 4.89 & 69.43 \\
\hline \multirow{3}{*}{ CG } & 0.185 & 50 & 9.27 & 1.91 & 9.4 & 1.7 & Qualified & 6.33 & 61.09 \\
\hline & 0.595 & 50 & 29.75 & 1.93 & 9.4 & 1.7 & Qualified & 5.70 & 70.68 \\
\hline & 0.355 & 25 & 8.87 & 1.85 & 9.3 & 1.6 & Qualified & 4.39 & 65.52 \\
\hline
\end{tabular}

${ }^{*} \mathrm{CV}=\mathrm{SD} /$ Mean $\times 100 \%$.

TABLE 2: Primers and product length of the four targeted genes.

\begin{tabular}{lcccc}
\hline No. & Gene symbol & Forward primer & Reverse primer & Product length \\
\hline 1 & $*$ ACTB & GCGTCCACCCGCGAGTACAA & ACATGCCGGAGCCGTTGTCG & 118 \\
2 & Irf7 & TGGCAGATGGAAGCTACC & GGCTATACAGGAACACGC & 154 \\
3 & Ninj2 & CCACCACCTTGGTCTTCATA & AGGCTGAAGTGGCTTTAG & 152 \\
4 & Iscal & CCCGTTGCATCTTTACCAC & GTCTAAGCAAACCGCATGAA & 151 \\
5 & Plxnc1 & TGACCACTGCCACTTGAT & CTGAAGAGTTTCTCAAGCAC & 159 \\
\hline
\end{tabular}

* refers to internal control gene.

74004, QIAGEN, GmBH, Germany) and RNase-Free DNase Set (Cat no. 79254, QIAGEN, GmBH, Germany) (Table 1).

2.7.2. RNA Amplification and Labeling. Total RNA was amplified and labeled by Low Input Quick Amp Labeling Kit, One-Color (Cat no. 5190-2305, Agilent technologies, Santa Clara, CA, US), following the manufacturer's instructions. Labeled cRNA were purified by RNeasy mini kit (Cat no. 74106, QIAGEN, GmBH, Germany).

2.7.3. Hybridization. Each slide was hybridized with $1.65 \mu \mathrm{g}$ Cy3-labeled cRNA using Gene Expression Hybridization Kit (Cat no. 5188-5242, Agilent technologies, Santa Clara, CA, US) in Hybridization Oven (Cat no. G2545A, Agilent technologies, Santa Clara, CA, US), according to the manufacturer's instructions. After 17 hours hybridization, slides were washed in staining dishes (Cat no. 121, Thermo Shandon, Waltham, MA, US) with Gene Expression Wash Buffer Kit (Cat no. 5188-5327, Agilent technologies, Santa Clara, CA, US), following the manufacturer's instructions.

2.7.4. Data Acquisition. Slides were scanned by Agilent Microarray Scanner (Cat no. G2565CA, Agilent technologies, Santa Clara, CA, US) with default settings: dye channel: Green, Scan resolution $=5 \mu \mathrm{m}$, PMT 100\%, 10\%, 16 bit. Feature Extraction software 10.7 (Agilent technologies, Santa Clara, CA, US) Raw data were normalized by Quantile algorithm, Gene Spring Software 11.0 (Agilent technologies, Santa Clara, CA, US) (Table 1).
2.7.5. Real-Time PCR. Primers of the four genes were designed with Primer Express 2.0 (Oebiotec, Shanghai, China) (Table 2). Reverse transcription was performed on PrimerScript RT reagent Kit (TaKaRa, DRR037A, Takara Biotechnology (Dalian) Co., Ltd. China). Total RNA $(0.5 \mu \mathrm{g})$ was denatured at room temperatrue then mixed with the reagent in a final volume of $10 \mu \mathrm{L}$ containing $50 \mu \mathrm{M}$ oligo dT, $100 \mu \mathrm{M}$ random primer, $0.5 \mathrm{mM} \mathrm{dNTP}$ and the manufacturer's buffer and Enzyme Mix. The RT reaction was conducted for $15 \mathrm{~min}$ at $37^{\circ} \mathrm{C}$, and $85^{\circ} \mathrm{C}$ for $5 \mathrm{~s}$ in ABI 9700. First-strand cDNA product was diluted in $100 \mu \mathrm{L}$ distilled water in preparation for real-time PCR. qPCR was performed using SuperReal PreMix (SYBR Green) kit (TIANGEN, FP204, Tiangen Biotech (Beijing) Co., Ltd. Beijing, China). Briefly, $1 \mu \mathrm{L}$ of diluted cDNA product was used for 40-cycle three-step PCR in a Roche HOLD CYCLE LightCycler 480 II.

2.8. Statistical Analysis. The body development, behavioral test, and hormone level data were analyzed using a Statistical Package for the Social Sciences (SPSS) version 19.0. ANOVA for Repeated Measurement with Greenhouse-Geisser Adjustment was performed to analyze group differences in body weight. A nonparametric Mann-Whitney test was performed to analyze group differences on the OFT. Student's $t$-test was performed to analyze group differences in corticosterone, dopamine, 5-HT, and growth hormone. Alpha was set to.05 for all analyses. 
TABLE 3: Differentially expressed genes in ESG versus CG, among which 39 genes were upregulated and 81 genes downregulated.

\begin{tabular}{|c|c|c|c|c|}
\hline Gene ID & $P$ values & Fold change & Gene symbol & Regulation \\
\hline 63847 & 0.007006 & 0.096204 & Fxyd6 & Downregulated \\
\hline 498145 & 0.003225 & 0.17368 & LOC498145 & Downregulated \\
\hline 316628 & 0.004414 & 0.274831 & Asb1 & Downregulated \\
\hline 360547 & 0.005836 & 0.320844 & Sat2 & Downregulated \\
\hline 301245 & 0.007067 & 0.331729 & Yipf3 & Downregulated \\
\hline 293023 & 0.009502 & 0.335662 & Klhl25 & Downregulated \\
\hline 288240 & 0.002174 & 0.344925 & Hlcs & Downregulated \\
\hline 293180 & 0.007695 & 0.352823 & Micalcl & Downregulated \\
\hline 316426 & 0.003961 & 0.363248 & Spats2l & Downregulated \\
\hline 293624 & 0.008043 & 0.364195 & Irf7 & Downregulated \\
\hline 683788 & 0.007907 & 0.382175 & LOC683788 & Downregulated \\
\hline 293156 & 0.009012 & 0.413953 & Lrtomt & Downregulated \\
\hline 25646 & 0.004102 & 0.429726 & Otx1 & Downregulated \\
\hline 290232 & 0.009311 & 0.430944 & Tinf2 & Downregulated \\
\hline 498353 & 0.002896 & 0.440115 & Scfd 2 & Downregulated \\
\hline 362873 & 0.006203 & 0.440433 & Plxnc1 & Downregulated \\
\hline 309415 & 0.009479 & 0.458925 & Fam189a2 & Downregulated \\
\hline 113894 & 0.007725 & 0.463149 & Sqstm1 & Downregulated \\
\hline 303538 & 0.003261 & 0.465171 & Dhx58 & Downregulated \\
\hline 406196 & 0.001118 & 0.467157 & $\mathrm{Hcr}$ & Downregulated \\
\hline 313917 & 0.005676 & 0.482298 & Abhd1 & Downregulated \\
\hline 292811 & 0.009904 & 0.48439 & Ccdc123 & Downregulated \\
\hline 290985 & 0.007918 & 0.491881 & Iscal & Downregulated \\
\hline 405152 & 0.008771 & 0.516648 & Olr1192 & Downregulated \\
\hline 171355 & 0.005274 & 0.519609 & Pou4f2 & Downregulated \\
\hline 362943 & 0.000172 & 0.526926 & Adck5 & Downregulated \\
\hline 309161 & 0.001612 & 0.543788 & Ccdc85b & Downregulated \\
\hline 361327 & 0.003693 & 0.596748 & Prr16 & Downregulated \\
\hline 24640 & 0.008865 & 0.602226 & Pfkfb2 & Downregulated \\
\hline 619573 & 0.006811 & 0.603084 & Fam104a & Downregulated \\
\hline 116725 & 0.007447 & 0.653258 & Ube2n & Downregulated \\
\hline 304342 & 0.005141 & 0.662423 & Zscan21 & Downregulated \\
\hline 192252 & 0.009069 & 0.671766 & Dctpp1 & Downregulated \\
\hline 114205 & 0.00295 & 0.677239 & Crcp & Downregulated \\
\hline 311430 & 0.007769 & 0.689602 & Mavs & Downregulated \\
\hline 287840 & 0.003671 & 0.716317 & Fam100b & Downregulated \\
\hline 297109 & 0.006823 & 0.764608 & MGC95152 & Downregulated \\
\hline 295037 & 0.000491 & 0.788096 & Mgst2 & Downregulated \\
\hline 100360990 & 0.007759 & 0.815928 & LOC100360990 & Downregulated \\
\hline 501083 & 0.00564 & 1.179002 & Pdcd6ip & Upregulated \\
\hline 299195 & 0.000513 & 1.189394 & Coq6 & Upregulated \\
\hline 81716 & 0.007768 & 1.20684 & $\operatorname{Ggcx}$ & Upregulated \\
\hline 315023 & 0.008157 & 1.265746 & Slc25a32 & Upregulated \\
\hline 296753 & 0.009238 & 1.284846 & Srpk2 & Upregulated \\
\hline 299147 & 0.005455 & 1.304917 & Ppp2r5e & Upregulated \\
\hline 361932 & 0.009554 & 1.307515 & RGD1561393 & Upregulated \\
\hline 288259 & 0.009614 & 1.31293 & Gart & Upregulated \\
\hline 289522 & 0.002341 & 1.325268 & Cox18 & Upregulated \\
\hline 50688 & 0.002132 & 1.334825 & Cacnb1 & Upregulated \\
\hline 363171 & 0.000593 & 1.337206 & Tmem42 & Upregulated \\
\hline 114215 & 0.005997 & 1.352079 & Insl3 & Upregulated \\
\hline
\end{tabular}


Table 3: Continued.

\begin{tabular}{|c|c|c|c|c|}
\hline Gene ID & $P$ values & Fold change & Gene symbol & Regulation \\
\hline 315771 & 0.008317 & 1.369011 & Herc1 & Upregulated \\
\hline 360389 & 0.009442 & 1.375028 & Zfp422 & Upregulated \\
\hline 305923 & 0.008185 & 1.393988 & Zdhhc20 & Upregulated \\
\hline 24803 & 0.005163 & 1.399617 & Vamp2 & Upregulated \\
\hline 363210 & 0.001697 & 1.411325 & Phf3 & Upregulated \\
\hline 50561 & 0.001722 & 1.425023 & Resp18 & Upregulated \\
\hline 362367 & 0.005441 & 1.43527 & Znrf2 & Upregulated \\
\hline 170841 & 0.009557 & 1.458549 & Mutyh & Upregulated \\
\hline 81678 & 0.003588 & 1.464706 & Itpr2 & Upregulated \\
\hline 502886 & 0.009395 & 1.466283 & Foxj2 & Upregulated \\
\hline 360868 & 0.009274 & 1.471063 & $\mathrm{Sft} 2 \mathrm{~d} 2$ & Upregulated \\
\hline 313757 & 0.005281 & 1.485264 & RGD1565591 & Upregulated \\
\hline 361109 & 0.000669 & 1.486251 & Dcpla & Upregulated \\
\hline 192210 & 0.008713 & 1.487999 & Dnajc21 & Upregulated \\
\hline 25262 & 0.008127 & 1.49478 & Itpr1 & Upregulated \\
\hline 311112 & 0.00906 & 1.533447 & Fastkd1 & Upregulated \\
\hline 64086 & 0.004012 & 1.55121 & Csnk1g1 & Upregulated \\
\hline 366693 & 0.007515 & 1.567923 & Rbm25 & Upregulated \\
\hline 690961 & 0.006894 & 1.577038 & $\operatorname{Cog} 2$ & Upregulated \\
\hline 292148 & 0.004257 & 1.589999 & Eif3a & Upregulated \\
\hline 691918 & 0.002531 & 1.596744 & LOC691918 & Upregulated \\
\hline 362317 & 0.001503 & 1.599092 & Krit1 & Upregulated \\
\hline 54323 & 0.001154 & 1.610286 & Arc & Upregulated \\
\hline 304813 & 0.005676 & 1.614358 & Ppp1r12b & Upregulated \\
\hline 58983 & 0.00216 & 1.617294 & Rabggta & Upregulated \\
\hline 361944 & 0.004739 & 1.617335 & Elf2 & Upregulated \\
\hline 314862 & 0.000215 & 1.618023 & Dyrk2 & Upregulated \\
\hline 29642 & 0.003006 & 1.62079 & Slc38a2 & Upregulated \\
\hline 291409 & 0.00357 & 1.622726 & Zfp236 & Upregulated \\
\hline 246282 & 0.001061 & 1.623318 & Zfp91 & Upregulated \\
\hline 362132 & 0.00226 & 1.626565 & Epc2 & Upregulated \\
\hline 303963 & 0.002236 & 1.631518 & Dzip3 & Upregulated \\
\hline 116670 & 0.006773 & 1.634179 & Ppp1r12a & Upregulated \\
\hline 302670 & 0.004529 & 1.63737 & Zrsr2 & Upregulated \\
\hline 360993 & 0.006601 & 1.637448 & Smek2 & Upregulated \\
\hline 59319 & 0.001208 & 1.6438 & Nyw1 & Upregulated \\
\hline 287249 & 0.009286 & 1.659325 & Cnot6 & Upregulated \\
\hline 362132 & 0.007917 & 1.663529 & Epc2 & Upregulated \\
\hline 303511 & 0.004368 & 1.665157 & Ikzf3 & Upregulated \\
\hline 363210 & 0.008478 & 1.665263 & Phf3 & Upregulated \\
\hline 362096 & 0.00268 & 1.668933 & Setx & Upregulated \\
\hline 316583 & 0.001117 & 1.700923 & B3gnt7 & Upregulated \\
\hline 362817 & 0.008175 & 1.701909 & $\mathrm{Cdk} 2$ & Upregulated \\
\hline 304157 & 0.009185 & 1.708222 & Nrip1 & Upregulated \\
\hline 314169 & 0.009008 & 1.729076 & Fam179b & Upregulated \\
\hline 303919 & 0.007784 & 1.731828 & Lrrc58 & Upregulated \\
\hline 309523 & 0.005447 & 1.734164 & Kif20b & Upregulated \\
\hline 291773 & 0.003136 & 1.741424 & RGD1562997 & Upregulated \\
\hline 314423 & 0.003545 & 1.743689 & Bcl11b & Upregulated \\
\hline 362622 & 0.007916 & 1.756522 & Ccdc21 & Upregulated \\
\hline 497198 & 0.005781 & 1.770803 & Impact & Upregulated \\
\hline
\end{tabular}


Table 3: Continued.

\begin{tabular}{|c|c|c|c|c|}
\hline Gene ID & $P$ values & Fold change & Gene symbol & Regulation \\
\hline 315804 & 0.00029 & 1.773739 & Rfx7 & Upregulated \\
\hline 363287 & 0.002339 & 1.775948 & Hdac4 & Upregulated \\
\hline 361688 & 0.00606 & 1.778637 & Suv420h1 & Upregulated \\
\hline 363555 & 0.002239 & 1.787221 & Wfikkn1 & Upregulated \\
\hline 304809 & 0.001337 & 1.791911 & $\mathrm{Kdm} 5 \mathrm{~b}$ & Upregulated \\
\hline 498803 & 0.003675 & 1.797804 & Otud1 & Upregulated \\
\hline 64624 & 0.005484 & 1.803225 & Cul5 & Upregulated \\
\hline 304817 & 0.00381 & 1.807047 & Ipo9 & Upregulated \\
\hline 54311 & 0.008729 & 1.82334 & Timm17a & Upregulated \\
\hline 25486 & 0.008651 & 1.8782 & Myo9b & Upregulated \\
\hline 302612 & 0.006615 & 1.978189 & Tspyl2 & Upregulated \\
\hline 293765 & 0.003013 & 2.076238 & Olr327 & Upregulated \\
\hline 171347 & 0.007854 & 2.324322 & Mat2a & Upregulated \\
\hline 685074 & 0.008629 & 2.417108 & LOC685074 & Upregulated \\
\hline 498211 & 0.007458 & 2.449546 & RGD1560523 & Upregulated \\
\hline 690043 & 0.004624 & 2.470614 & Rnf168 & Upregulated \\
\hline 171347 & 0.00179 & 2.47901 & Mat2a & Upregulated \\
\hline 363083 & 0.007379 & 2.521284 & Fbxl22 & Upregulated \\
\hline
\end{tabular}

\section{Results}

3.1. Body Development and Behavior Test. ANOVA for Repeated Measurement with Greenhouse-Geisser Adjustment (Mauchly's $W=0.085$, Approx. Chi-square $=214.490$, $\mathrm{df}=14, P \leqslant 0.001$, Greenhouse-Geisser $=0.541)$ showed a statistically significant difference of the body weight of the 6 observation time spots of offspring among CG, ESG, and HG offspring (body weight: $\mathrm{df}=2.705$, mean square $=$ 39791.256, $F=1923.553, P \leqslant 0.001$; body weight $*$ group $\mathrm{df}=5.410$, mean square $=415.400, F=20.081$, $P \leqslant 0.001)$. Generally, HG offspring was heavier than CG, which is heavier than ESG (Figure 1).

A Mann-Whitney test showed significant difference between the three groups on the OFT (Mann-Whitney $U=$ 1448.500, Wilcoxon $W=2529.500, Z=-3.819, P=$ 0.000) (Figure 2): the OFT scores of HG and CG were both significantly higher than those observed in the ESG.

3.2. Hormone Levels. The corticosterone levels of CG was statistically lower than ESG and slightly than HG (Figure 3(a)). The dopamine level of ESG was slightly lower than the CG and of HG was significantly higher than the ESG (Figure 3(b)). The 5-HT of ESG showed a highest level and the CG lowest (Figure 3(c)). The growth hormone level of the HG was statistically higher than the CG and ESG (Figure 3(d)).

\subsection{Gene Expression Profile}

3.3.1. ESG versus CG. Gene expression profile showed 81 genes upregulated and 39 genes downregulated $(P<0.01)$ in ESG versus CG comparison (Table 3 (see Supporting
Information 1), Figure 4), among which 14 GO annotations were obtained including, ligase activity, regulation of metabolic process, positive regulation of metabolic process, cellular component assembly, membrane bounded organelle, biosynthetic process, cellular component biogenesis, and cellular response to stimulus. (Table 4 (Supporting Information 2)), and among which 12 KEGG pathways were annotated, including oocyte meiosis, vascular smooth muscle contraction, RIG-I-like receptor signaling pathway, longterm potentiation, ubiquitin mediated proteolysis, and longterm depression (Table 5).

3.3.2. ESG versus $H G$. Gene expression profile showed 60 genes upregulated and 28 genes downregulated $(P<0.01)$ in ESG versus CG (Table 6 (Supporting Information 3), Figure 5), among which five GO annotations were obtained including protein complex localization, cellular component assembly, cellular component biogenesis, anatomical structure formation, and organelle lumen (Table 7), and among which 5 KEGG pathways were annotated, including cell cycle, Jak-STAT signaling pathway, Type II diabetes mellitus, One carbon pool by folate, and insulin signaling pathway (Table 8).

No genes were found, which were significantly differently expressed simultaneously in ESG versus CG and ESG versus HG. However, 8,426 genes were found no statistical difference in HG versus CG $(P>0.05)$ among which 84 were found also presented in the differently expressed genes in ESG versus HG (Table 9 (Supporting Information 4)).

3.3.3. RT-PCR Validation. Irf7, Ninj2, Plxnc1, and Isca1 were filtered to validate with RT-PCR according to the set that the flag value of the expression profile chip $\neq A, \mathrm{FC}>$ 2 or $\mathrm{FC}<0.5$, expression value $\geq 6$ from the GO and 
TABle 4: Significant GO annotation of the 120 differentially expressed genes and the genes involved $(P<0.05)$.

\begin{tabular}{|c|c|c|c|c|c|c|}
\hline GO Id & Name & Symbol & Hits & Total & Percent & $\begin{array}{l}\text { Enrichment } \\
\text { test } P \text { value }\end{array}$ \\
\hline \multirow{3}{*}{ GO: 0016874} & \multirow{3}{*}{ Ligase activity } & Ube2n, Hlcs & \multirow{3}{*}{7} & \multirow{3}{*}{308} & \multirow{3}{*}{$2.27 \%$} & \multirow{3}{*}{0.0083} \\
\hline & & Gart, Herc1, & & & & \\
\hline & & Cul5, Rnf168, Ggcx & & & & \\
\hline \multirow{7}{*}{ GO: 0019222} & \multirow{7}{*}{$\begin{array}{l}\text { Regulation of } \\
\text { metabolic } \\
\text { process }\end{array}$} & Sqstm1, Insl3, Ube2n, Pou4f2, & \multirow{7}{*}{28} & \multirow{7}{*}{2415} & \multirow{7}{*}{$1.16 \%$} & \multirow{7}{*}{0.0089} \\
\hline & & Otx1, Cnot6, Tinf2, & & & & \\
\hline & & RGD1562997, Irf7, & & & & \\
\hline & & Tspyl2, Nrip1, & & & & \\
\hline & & Zscan21, Jarid1b, Bcl11b, Dyrk2, & & & & \\
\hline & & $\begin{array}{l}\text { Mll1, Rfx7, Zfp422, Smek2, Suv420h1, Elf2, } \\
\text { Cdk2, Hdac4, Impact, }\end{array}$ & & & & \\
\hline & & Foxj2, Rasd1, Rnf168, Pfn2 & & & & \\
\hline \multirow{5}{*}{ GO: 0009893} & \multirow{5}{*}{$\begin{array}{l}\text { Positive } \\
\text { regulation of } \\
\text { metabolic } \\
\text { process }\end{array}$} & Sqstm1, Insl3, Ube2n, & \multirow{5}{*}{13} & \multirow{5}{*}{846} & \multirow{5}{*}{$1.54 \%$} & \multirow{5}{*}{0.0098} \\
\hline & & Pou4f2, Tinf2, & & & & \\
\hline & & Nrip1, Zscan21, Bcl11b & & & & \\
\hline & & Dyrk2, Mll1, Cdk2 & & & & \\
\hline & & Hdac4, Rnf168 & & & & \\
\hline \multirow{3}{*}{ GO: 0022607} & \multirow{3}{*}{$\begin{array}{l}\text { Cellular } \\
\text { component } \\
\text { assembly }\end{array}$} & Sqstm1, Xtp3tpa & \multirow{3}{*}{12} & \multirow{3}{*}{786} & \multirow{3}{*}{$1.53 \%$} & \multirow{3}{*}{0.0135} \\
\hline & & Vamp2, Cox18, Tinf2, Eif3s10, RGD1562997 & & & & \\
\hline & & Srpk2, Mll1, Enth, Pfn2 & & & & \\
\hline \multirow{14}{*}{ GO: 0043227} & \multirow{14}{*}{$\begin{array}{l}\text { Membrane- } \\
\text { bounded } \\
\text { organelle }\end{array}$} & Sqstm1, Crcp, Ube2n, Mutyh, Pou4f2, Vamp2 & \multirow{14}{*}{55} & \multirow{14}{*}{5982} & \multirow{14}{*}{$0.92 \%$} & \\
\hline & & Itpr1, Otx1, Cnot6 & & & & \\
\hline & & Hlcs, Cox18, Tinf2 & & & & \\
\hline & & Iscal, Eif3s10 & & & & \\
\hline & & RGD1562997 & & & & \\
\hline & & Irf7, Srpk2, Ikzf3 & & & & \\
\hline & & Ppp2r5e, Yipf3 & & & & \\
\hline & & Tspyl2, Zrsr2, Nrip1 & & & & \\
\hline & & Zscan21, Kif20b & & & & \\
\hline & & Visa, RGD1565591 & & & & 0.025 \\
\hline & & Bcl11b, Dyrk2 & & & & \\
\hline & & Slc25a32, Mll1, Enth & & & & \\
\hline & & B3gnt7, Zfp422, Setx & & & & \\
\hline & & Suv420h1, Elf2, Phf3 & & & & \\
\hline
\end{tabular}


Table 4: Continued.

\begin{tabular}{|c|c|c|c|c|c|c|}
\hline GO Id & Name & Symbol & Hits & Total & Percent & $\begin{array}{l}\text { Enrichment } \\
\text { test } P \text { value }\end{array}$ \\
\hline & & Cdk2, Adck5, Hdac4 Hcr, LOC498145 & & & & \\
\hline & & Pdcd6ip, Foxj2, Rasd1, Resp18, Cul5 & & & & \\
\hline & & Cacnb1,Timm17a, & & & & \\
\hline & & Arc, Rnf168, Cog2, & & & & \\
\hline & & Itpr2, Ggcx & & & & \\
\hline GO: 0014854 & $\begin{array}{l}\text { Response to } \\
\text { inactivity }\end{array}$ & Hdac4 & 1 & 3 & $33.33 \%$ & 0.0288 \\
\hline \multirow{12}{*}{ GO: 0009058} & \multirow{12}{*}{$\begin{array}{l}\text { Biosynthetic } \\
\text { process }\end{array}$} & Crcp, Insl3,Ube2n & & & & \\
\hline & & Mat2a, Pou4f2, Otx1 & & & & \\
\hline & & Cnot6, Gart, Tinf2 & & & & \\
\hline & & Isca1, RGD1562997 & & & & \\
\hline & & Eif3s10, Irf7, Coq6 & & & & \\
\hline & & Tspyl2, Nrip1, Mll1 & 34 & 3379 & $1.01 \%$ & 0.0291 \\
\hline & & Zscan21, Jarid1b & & & & \\
\hline & & Bcl11b, Dyrk2, Rfx7 & & & & \\
\hline & & B3gnt7, Zfp422, Elf2 & & & & \\
\hline & & Suv420h1, Cdk2, Phf3 & & & & \\
\hline & & Hdac4, Impact, Foxj2 & & & & \\
\hline & & Rabggta, Rasd 1 & & & & \\
\hline \multirow{4}{*}{ GO: 0044085} & \multirow{4}{*}{$\begin{array}{l}\text { Cellular } \\
\text { component } \\
\text { biogenesis }\end{array}$} & Sqstm1, Xtp3tpa, & & & & \\
\hline & & Vamp2, Cox18, Tinf2 & & & & \\
\hline & & RGD1562997, Eif3s10 & 12 & 883 & $1.36 \%$ & 0.0299 \\
\hline & & Srpk2, Mll1, Enth, Pfn2 & & & & \\
\hline GO: 0014874 & $\begin{array}{l}\text { Response to } \\
\text { stimulus } \\
\text { involved in } \\
\text { regulation of } \\
\text { muscle } \\
\text { adaptation }\end{array}$ & Hdac4 & 1 & 4 & $25.00 \%$ & 0.0359 \\
\hline \multirow{6}{*}{ GO: 0043233} & \multirow{6}{*}{$\begin{array}{l}\text { Organelle } \\
\text { lumen }\end{array}$} & Sqstm1, Mutyh, Itpr1 & & & & \\
\hline & & Tinf2, RGD1562997 & & & & \\
\hline & & Srpk2, Tspyl2, Zrsr2 & 16 & 1360 & $1.18 \%$ & 0.0416 \\
\hline & & Nrip1, Kif20b, Mll1 & & & & \\
\hline & & Zfp422, Setx, Cdk2 & & & & \\
\hline & & Hdac4, Resp18 & & & & \\
\hline GO: 0051716 & $\begin{array}{l}\text { Cellular } \\
\text { response to } \\
\text { stimulus }\end{array}$ & $\begin{array}{l}\text { Ube2n, Mutyh, Dyrk2 } \\
\text { Mll1, Setx, Cdk2, }\end{array}$ & 8 & 528 & $1.52 \%$ & 0.0422 \\
\hline
\end{tabular}


TABle 4: Continued.

\begin{tabular}{|c|c|c|c|c|c|c|}
\hline GO Id & Name & Symbol & Hits & Total & Percent & $\begin{array}{l}\text { Enrichment } \\
\text { test } P \text { value }\end{array}$ \\
\hline & & Pdcd6ip, Rnf168 & & & & \\
\hline \multirow{6}{*}{ GO: 0016740} & \multirow{6}{*}{$\begin{array}{l}\text { Transferase } \\
\text { activity }\end{array}$} & Crcp, Mat2a, Pfkfb2 & \multirow{6}{*}{18} & \multirow{6}{*}{1612} & \multirow{6}{*}{$1.12 \%$} & \multirow{6}{*}{0.0483} \\
\hline & & Gart, Mgst2, Srpk2 & & & & \\
\hline & & RGD1304822, Dyrk2 & & & & \\
\hline & & Fastkd1, Mll1, B3gnt7 & & & & \\
\hline & & Suv420h1, Cdk2, Fgfr1l, RGD1560523 & & & & \\
\hline & & Rabggta, Csnk1g1 & & & & \\
\hline \multirow{6}{*}{ GO: 0031974} & \multirow{6}{*}{$\begin{array}{l}\text { Membrane } \\
\text { enclosed lumen }\end{array}$} & Sqstm1, Mutyh, Itpr1 & \multirow{6}{*}{16} & \multirow{6}{*}{1392} & \multirow{6}{*}{$1.15 \%$} & \multirow{6}{*}{0.0495} \\
\hline & & Tinf2, RGD1562997 & & & & \\
\hline & & Srpk2, Tspyl2, Zrsr2 & & & & \\
\hline & & Nrip1, Kif20b, Mll1 & & & & \\
\hline & & Zfp422, Setx, Cdk2, & & & & \\
\hline & & Hdac4, Resp18 & & & & \\
\hline GO: 0031077 & $\begin{array}{l}\text { Postembryonic } \\
\text { camera-type eye } \\
\text { development }\end{array}$ & Bcl11b & 1 & 6 & $16.67 \%$ & 0.0499 \\
\hline
\end{tabular}

KEGG annotation. As showed in Figure 6(a), Irf7, Ninj2, and Iscal were significantly hypoexpressed in ESG $(\mathrm{FC}<0.5)$; however, the gene expression of Plxncl did not match the RT-PCR validation; in Figure 6(b), the four genes were not significantly hypoexpressed in $\mathrm{HG}$ versus $\mathrm{CG}(0.5<\mathrm{FC}<2)$, and the RT-PCR validation showed an obviously reduced $\Delta \Delta \mathrm{Ct}$ values compared with those in Figure 6(a). The gene expression profile chip outcomes showed a favorable match with the RT-PCR result.

\section{Discussion}

Substantial evidence from preclinical laboratory studies indicates that PS affects the hormonal and behavioral development of offspring. PS has been found to alter baseline and stress-induced responsivity of the HPA axis and levels and distribution of regulatory neurotransmitters, such as norepinepherine, dopamine, serotonin, and acetylcholine and to modify key limbic structures and to retard intrauterine growth [15]. In this study, ESG demonstrated differences from CG on body weight, hormone levels, and gene expressions, and HG differed from the ESG group on body weight, hormone levels, and gene expressions. From the perspective of Chinese medicine, once parental kidney is injured from PS, manifestations are handed down to offspring, showing development retardation and OFT performance reduction. JKSQW is a typical herbal formula for kidney qi supplementing, which recovers the physiological functions of kidney. In this study, the body weight and OFT performance were improved by JKSQW, supporting the effectiveness of Chinese herb remedy in rodents in lab [13].

Experimentally, PS in animal models mal-programs offspring physiology, resulting in increasing the likelihood of disorders of HPA axis activity and anxiety-related behaviors in adulthood [16]. PS increases plasma levels of corticosterone and corticotrophin releasing hormone in the mother and fetus, which may contribute to insulin resistance and behavior disorders in their offspring that include attention and learning deficits, generalized anxiety and depression [17]. We demonstrated that the serum corticosterone of ESG were significantly higher than CG and slightly higher than HG, which was in accordance with previous reports [1820]. Animal studies indicate that PS can affect the activity of the placental barrier enzyme 11- $\beta$ HSD2 (11 $\beta$-hydroxysteroid dehydrogenase type 2), which metabolizes corticosterone $[2,17]$. 5-HT level of ESG was significantly higher than CG and HG. Alterations in activity of the central 5-HT system play an essential role in many of these behavioral aberrations due to PS $[21,22]$. During pregnancy, the 5-HT system has a fundamental role in the fetus' development of the central nervous system, and 5-HT neurotransmission is involved in the activation and feedback of HPA axis throughout life [23]. Huang et al. [14] reported that levels of 5-HT were higher in rat hippocampus and hypothalamus of fetuses in the CUS group, that is, chronic unpredictable stress maternally performed than in the controls. Increased 5-HT signaling increases the expression of key transcription factors, notably 
TAble 5: KEGG Pathway annotation of the 120 differentially expressed genes $(P<0.05, q<0.05)(\downarrow$ refers downregulation, $\uparrow$ refers upregulation).

\begin{tabular}{|c|c|c|c|c|c|}
\hline Name & Symbol & Total & Percent & $\begin{array}{l}\text { Enrichment } \\
\text { test } P \text { value }\end{array}$ & $q$ value \\
\hline \multirow[t]{2}{*}{ Oocyte meiosis } & 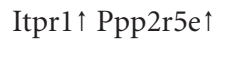 & 116 & 0.0345 & 0.0008 & 0.0048 \\
\hline & $\mathrm{Cdk} 2 \uparrow$ & & & & \\
\hline \multirow{3}{*}{$\begin{array}{l}\text { Vascular smooth muscle } \\
\text { contraction }\end{array}$} & Ppp1r12a & & & & \\
\hline & Itpr $1 \uparrow$ & 128 & 0.0313 & 0.0011 & 0.0048 \\
\hline & Ppplr12b $\uparrow$ & & & & \\
\hline \multirow{2}{*}{$\begin{array}{l}\text { RIG-I-like receptor signaling } \\
\text { pathway }\end{array}$} & Irf7 $\downarrow$ Dhx58 & 64 & 0.0469 & 0.0016 & 0.0048 \\
\hline & Mavs $\downarrow$ & & & & \\
\hline \multirow[t]{2}{*}{ Long-term potentiation } & Ppp1r12a $\uparrow \operatorname{Itpr} 1 \uparrow$ & 72 & 0.0417 & 0.0022 & 0.0049 \\
\hline & Itpr $2 \uparrow$ & & & & \\
\hline \multirow[t]{2}{*}{ Ubiquitin mediated proteolysis } & Ube2n $\downarrow$ Herc $1 \uparrow$ & 132 & 0.0227 & 0.0111 & 0.0176 \\
\hline & 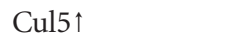 & & & & \\
\hline Cytosolic DNA-sensing pathway & Irf7 $\downarrow$ Mavs $\downarrow$ & 49 & 0.0408 & 0.0131 & 0.0176 \\
\hline Biotin metabolism & Hlcs $\downarrow$ & 3 & 0.3333 & 0.0135 & 0.0176 \\
\hline RNA degradation & Cnot6 $\uparrow$ Dcpla $\uparrow$ & 61 & 0.0328 & 0.0196 & 0.0223 \\
\hline Long-term depression & Itpr $1 \uparrow \operatorname{Itpr} 2 \uparrow$ & 69 & 0.029 & 0.0245 & 0.0245 \\
\hline $\begin{array}{l}\text { Ubiquinone and other } \\
\text { terpenoid-quinone biosynthesis }\end{array}$ & Coq6i & 7 & 0.1429 & 0.0269 & 0.0245 \\
\hline $\begin{array}{l}\text { Phosphatidylinositol signaling } \\
\text { system }\end{array}$ & Itpr $2 \uparrow \operatorname{Itpr} 1 \uparrow$ & 77 & 0.026 & 0.0299 & 0.0247 \\
\hline Gap junction & Itpr $2 \uparrow \operatorname{Itpr} 1 \uparrow$ & 87 & 0.023 & 0.0371 & 0.0281 \\
\hline GnRH signaling pathway & Itpr $1 \uparrow \operatorname{Itpr} 2 \uparrow$ & 99 & 0.0202 & 0.0467 & 0.0326 \\
\hline
\end{tabular}

nerve growth factor induced protein $\mathrm{A}$, which binds to and regulates activation of the GR promoter [24]. No difference of the dopamine level between ESG and CG were obtained, indicating earthquake may not alter the offspring dopamine. Interestingly JKSQW in HG significantly elevated the dopamine level of ESG. Carboni et al. [25] reported prenatal catecholamine stimulation was obtained by amphetamine or nicotine. We observed that PS did not change dopamine. No difference of the hormone level between ESG and CG were obtained, indicating earthquake may not impact on the growth hormone of offspring. Interestingly, however, JKSQW in HG significantly elevated the dopamine level of ESG, which might be explained by the function of kidney that governs development. Shen and Cai [26] reported that growth hormone genes were downregulated in a kidney-qi deficiency rat model and Chinese formula supplementing kidney qi could correct the downregulation. Mak et al. [27] found that chronic kidney disease in children was 
TABLE 6: Differentially expressed genes in ESG versus HG, among which 60 genes were upregulated and 28 genes downregulated.

\begin{tabular}{|c|c|c|c|c|}
\hline Gene ID & $P$ values & Fold change & Symbol & Remark \\
\hline 287881 & 0.006042 & 0.220799 & Dysfip 1 & Downregulated \\
\hline 25405 & 0.004824 & 0.344631 & Ccng1 & Downregulated \\
\hline 24237 & 0.003207 & 0.40894 & C6 & Downregulated \\
\hline 313219 & 0.003811 & 0.410283 & Zfp189 & Downregulated \\
\hline 287343 & 0.008194 & 0.499299 & Olr1454 & Downregulated \\
\hline 293156 & 0.008272 & 0.508908 & Lrtomt & Downregulated \\
\hline 405143 & 0.009972 & 0.5345 & Olr803 & Downregulated \\
\hline 116724 & 0.000512 & 0.546672 & Epb4.113 & Downregulated \\
\hline 313917 & 0.00383 & 0.578297 & Abhd1 & Downregulated \\
\hline 83681 & 0.004251 & 0.581219 & Cish & Downregulated \\
\hline 301346 & 0.007628 & 0.609505 & Sema4c & Downregulated \\
\hline 315346 & 0.003519 & 0.619843 & Itga5 & Downregulated \\
\hline 56825 & 0.009009 & 0.625224 & Cym & Downregulated \\
\hline 690810 & 0.007066 & 0.637375 & Adat1 & Downregulated \\
\hline 313982 & 0.009162 & 0.653927 & RGD1561890 & Downregulated \\
\hline 363285 & 0.004745 & 0.660307 & Scly & Downregulated \\
\hline 316090 & 0.003533 & 0.683347 & Fam198a & Downregulated \\
\hline 24513 & 0.003494 & 0.687818 & Ivd & Downregulated \\
\hline 303384 & 0.007792 & 0.703077 & Mmp28 & Downregulated \\
\hline 246074 & 0.009445 & 0.718762 & Scd1 & Downregulated \\
\hline 500011 & 0.008188 & 0.726294 & RGD1563091 & Downregulated \\
\hline 362943 & 0.004839 & 0.735253 & Adck 5 & Downregulated \\
\hline 500420 & 0.008119 & 0.744282 & LOC500420 & Downregulated \\
\hline 399489 & 0.006413 & 0.763541 & E2f1 & Downregulated \\
\hline 311716 & 0.004912 & 0.77549 & Col20a1 & Downregulated \\
\hline 113894 & 0.007846 & 0.78406 & Sqstm1 & Downregulated \\
\hline 266609 & 0.005228 & 0.798742 & Bles03 & Downregulated \\
\hline 246766 & 0.00514 & 0.821038 & Ggtal & Downregulated \\
\hline 288518 & 0.008613 & 1.136098 & RGD1311660 & Upregulated \\
\hline 499430 & 0.008063 & 1.148146 & Lrrc20 & Upregulated \\
\hline 317399 & 0.000156 & 1.156541 & Ddx21 & Upregulated \\
\hline 306182 & 0.00808 & 1.160148 & Ipo5 & Upregulated \\
\hline 301038 & 0.00729 & 1.178184 & Ubp1 & Upregulated \\
\hline 310806 & 0.006399 & 1.178549 & Cdc14a & Upregulated \\
\hline 287954 & 0.003091 & 1.181263 & Dgcr8 & Upregulated \\
\hline 260321 & 0.008611 & 1.181875 & Fkbp4 & Upregulated \\
\hline 305828 & 0.006609 & 1.182203 & Socs 4 & Upregulated \\
\hline 64161 & 0.005932 & 1.183779 & Pi4ka & Upregulated \\
\hline 290679 & 0.009165 & 1.186593 & Ints 10 & Upregulated \\
\hline 298429 & 0.006198 & 1.188777 & Rad54l & Upregulated \\
\hline 474154 & 0.005077 & 1.190852 & Rbm4b & Upregulated \\
\hline 288717 & 0.006268 & 1.196619 & Srrd & Upregulated \\
\hline 296312 & 0.004568 & 1.197256 & RGD1311066 & Upregulated \\
\hline 312640 & 0.005739 & 1.198178 & Tmem111 & Upregulated \\
\hline 83624 & 0.009311 & 1.200882 & Ppig & Upregulated \\
\hline 288778 & 0.001749 & 1.22319 & $\mathrm{~Pa} 2 \mathrm{~g} 4$ & Upregulated \\
\hline 362851 & 0.004166 & 1.224723 & $\mathrm{Cd} 320$ & Upregulated \\
\hline 308404 & 0.006579 & 1.227818 & $\operatorname{Irf} 2 b p 1$ & Upregulated \\
\hline 363760 & 0.005704 & 1.237527 & Arl6 & Upregulated \\
\hline 296076 & 0.007529 & 1.238081 & Srp14 & Upregulated \\
\hline 291787 & $6.57 E-05$ & 1.242186 & Rbbp8 & Upregulated \\
\hline
\end{tabular}


Table 6: Continued.

\begin{tabular}{|c|c|c|c|c|}
\hline Gene ID & $P$ values & Fold change & Symbol & Remark \\
\hline 500727 & 0.00344 & 1.246021 & Cdca4 & Upregulated \\
\hline 306587 & 0.008906 & 1.255527 & Tcta & Upregulated \\
\hline 29541 & 0.000917 & 1.259108 & Nthl1 & Upregulated \\
\hline 360855 & 0.004605 & 1.26267 & Smg7 & Upregulated \\
\hline 362317 & 0.008649 & 1.284527 & Krit1 & Upregulated \\
\hline 313757 & 0.004801 & 1.294664 & RGD1565591 & Upregulated \\
\hline 499370 & 0.009663 & 1.326682 & Itprip & Upregulated \\
\hline 288259 & 0.009472 & 1.335197 & Gart & Upregulated \\
\hline 29704 & 0.002213 & 1.349013 & Pacsin 1 & Upregulated \\
\hline 84472 & 0.006393 & 1.366251 & Ilf3 & Upregulated \\
\hline 363210 & 0.006023 & 1.388566 & Phf3 & Upregulated \\
\hline 680451 & 0.005563 & 1.419061 & Nrbp2 & Upregulated \\
\hline 311112 & 0.001699 & 1.426768 & Fastkd1 & Upregulated \\
\hline 54323 & 0.001608 & 1.4509 & Arc & Upregulated \\
\hline 309136 & 0.006405 & 1.452428 & Oraov1 & Upregulated \\
\hline 363169 & 0.005748 & 1.472567 & Toag1 & Upregulated \\
\hline 29642 & 0.004937 & 1.475875 & Slc38a2 & Upregulated \\
\hline 305461 & 0.004104 & 1.475879 & Fam53a & Upregulated \\
\hline 304813 & 0.00934 & 1.481691 & Ppp1r12b & Upregulated \\
\hline 680006 & 0.007932 & 1.484512 & Mad111 & Upregulated \\
\hline 304474 & 0.001635 & 1.497221 & Pitpnm2 & Upregulated \\
\hline 115768 & 0.009088 & 1.509009 & Zfp37 & Upregulated \\
\hline 301513 & 0.001268 & 1.512431 & Rqcd1 & Upregulated \\
\hline 363273 & 0.009331 & 1.521116 & Cops7b & Upregulated \\
\hline 293511 & 0.008749 & 1.533752 & Znf688 & Upregulated \\
\hline 245966 & 0.004372 & 1.544613 & Tmem150a & Upregulated \\
\hline 291409 & 0.003844 & 1.552189 & Zfp236 & Upregulated \\
\hline 84607 & 0.007931 & 1.552588 & Socs 2 & Upregulated \\
\hline 306344 & 0.007778 & 1.569477 & Arrdc2 & Upregulated \\
\hline 309828 & 0.006302 & 1.584851 & Tspyl4 & Upregulated \\
\hline 501095 & 0.009284 & 1.589281 & Rftn1 & Upregulated \\
\hline 81531 & 0.008017 & 1.606129 & Pfn2 & Upregulated \\
\hline 293152 & 0.007896 & 1.613085 & Art2b & Upregulated \\
\hline 497040 & 0.006162 & 1.71037 & Prss36 & Upregulated \\
\hline 171454 & 0.009816 & 1.850404 & Naccl & Upregulated \\
\hline 363827 & 0.00216 & 1.948295 & LOC363827 & Upregulated \\
\hline 364361 & 0.001905 & 4.479744 & RGD1563700 & Upregulated \\
\hline
\end{tabular}

Table 7: Significant GO Annotation of the 5 differentially expressed genes and the genes included $(P<0.05)$.

\begin{tabular}{|c|c|c|c|c|c|c|}
\hline GO ID & Name & Symbol & Hits & Total & Percent & $\begin{array}{l}\text { Enrichment } \\
\text { test } P \text { value }\end{array}$ \\
\hline GO: 0031503 & $\begin{array}{l}\text { Protein complex } \\
\text { localization }\end{array}$ & Fkbp4 & 1 & 5 & $20.00 \%$ & 0.0309 \\
\hline GO: 0022607 & $\begin{array}{l}\text { Cellular component } \\
\text { assembly }\end{array}$ & $\begin{array}{l}\text { Sqstm1, Nacc1, Ivd, Fkbp4, } \\
\text { Tspyl4, Itga5, Pfn2 }\end{array}$ & 8 & 786 & $1.02 \%$ & 0.0548 \\
\hline GO: 0044085 & $\begin{array}{l}\text { Cellular component } \\
\text { biogenesis }\end{array}$ & $\begin{array}{l}\text { Sqstm1, Nacc1, Ivd, Fkbp4, } \\
\text { Tspyl4, Itga5, Pfn2 }\end{array}$ & 8 & 883 & $0.91 \%$ & 0.0926 \\
\hline GO: 0010926 & $\begin{array}{l}\text { Anatomical structure } \\
\text { formation }\end{array}$ & $\begin{array}{l}\text { Sqstm1, Nacc1, Ivd, Fkbp4, } \\
\text { Ubp1, Tspyl4, Itga5, Pfn2 }\end{array}$ & 9 & 1049 & $0.86 \%$ & 0.0993 \\
\hline GO: 0043233 & Organelle lumen & $\begin{array}{l}\text { Sqstm1, Nacc1, Ivd, Fkbp4, } \\
\text { Pa2g4, Ints10, Nthl1, } \\
\text { Ddx21, E2f1, Rbm4b, Ppig }\end{array}$ & 11 & 1360 & $0.81 \%$ & 0.0994 \\
\hline
\end{tabular}


TABle 8: KEGG Pathway annotation of the 120 differentially expressed genes $(P<0.05, q<0.05)(\downarrow$ refers downregulation, $\uparrow$ refers upregulation).

\begin{tabular}{|c|c|c|c|c|c|}
\hline Name & Symbol & Total & Percent & Enrichment test $P$ value & $q$ value \\
\hline Cell cycle & $\begin{array}{c}\mathrm{Cdc} 14 \mathrm{a} \uparrow \\
\mathrm{E} 2 \mathrm{f} 1 \downarrow \\
\operatorname{Mad} 111 \uparrow\end{array}$ & 132 & 0.0227 & 0.0044 & 0.0067 \\
\hline Jak-STAT signaling pathway & $\begin{array}{c}\text { Socs } 4 \uparrow \\
\text { Cish } \downarrow \\
\text { Socs } 2 \uparrow\end{array}$ & 149 & 0.0201 & 0.0062 & 0.0067 \\
\hline Type II diabetes mellitus & $\begin{array}{l}\text { Socs } 4 \uparrow \\
\text { Socs } 2 \uparrow\end{array}$ & 53 & 0.0377 & 0.008 & 0.0067 \\
\hline One carbon pool by folate & Gart $\uparrow$ & 17 & 0.0588 & 0.0429 & 0.0158 \\
\hline Insulin signaling pathway & $\begin{array}{l}\text { Socs } 4 \uparrow \\
\text { Socs } 2 \uparrow\end{array}$ & 140 & 0.0143 & 0.0471 & 0.0158 \\
\hline
\end{tabular}

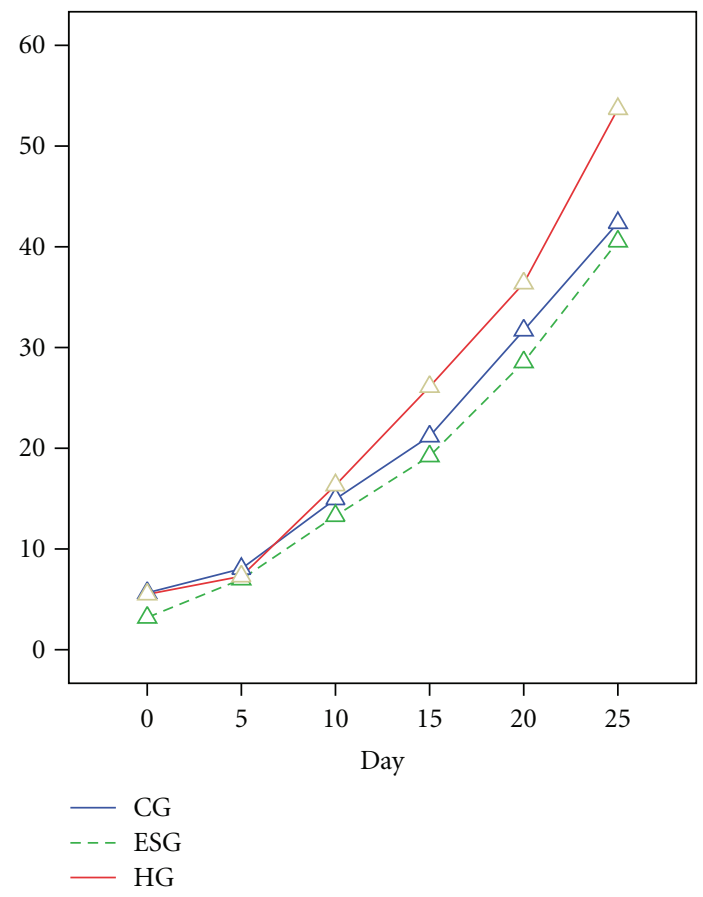

FIGURe 1: Mean plot of body weight. According to the ANOVA for Repeated Measurement, the body weight of ESG offspring were statistically all inferior to the CG offspring despite in Day $10(P<$ $0.05)$. The body weight HG offspring were statistically superior to the ESG offspring despite in Day $5(P<0.05)$; The body weight HG in Day 15, Day 20 and Day 25 were statistically superior to the CG $(P<0.05)$.

associated with dramatic changes in the growth hormone and insulin-like growth factor (IGF-1) axis, resulting in growth retardation. Yang and Li [28] reported that JKSQW could recover the downregulated growth hormone genes (Somatotropin precursor, NM-008117) in a kidney-yang deficiency rat model. Researches of the neurobiological

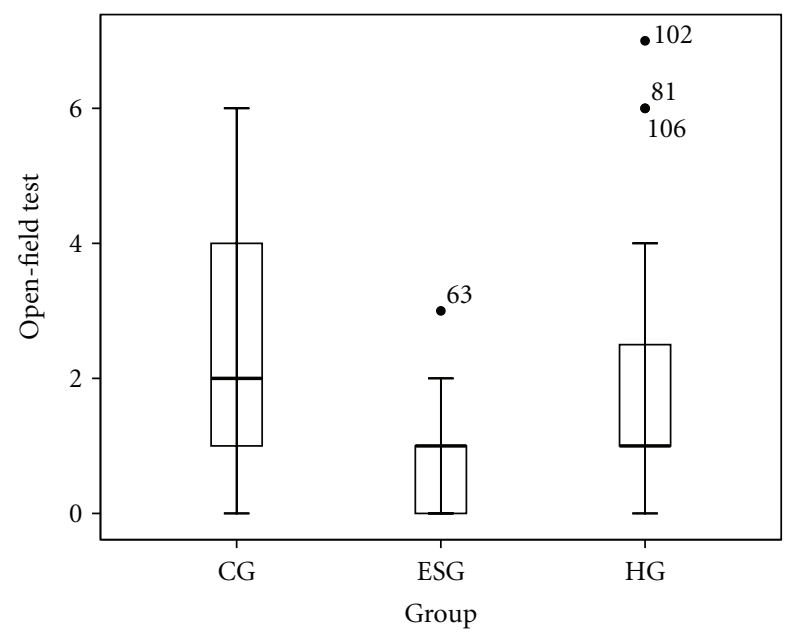

FIGURE 2: Box plot of OFT in the comparison between CG, ESG, and HG. ESG showed less scores than CG $(P<0.05)$ and HG $(P<$ 0.05).

mechanisms underlying the interaction between PS and adult mental disorders suggest the involvement of multiple neurotransmitter systems $[29,30]$. Findings of the hormones alterations suggest manual earthquake is a liable model modulating the fear from natural earthquake involving development retardation and neurotransmitter systems disorder. Meanwhile, from the perspective of Chinese medicine, kidney function is disturbed by the earthquake and recovered by JKSQW.

We found 81 genes upregulated and 39 genes downregulated in ESG versus CG, from which 14 significant GO and 12 KEGG pathways were annotated, indicating diversified and complicated physiological and psychological impacts on offspring left by the prenatal earthquake as a prenatal stress, for example, long-term depression and long-term potentiation. Mychasiuk et al. [31] reported that significant gene expression level changes in 558 different 
TABLE 9: The 84 genes differently expressed in ESG and normalized in HG (the $P$ value and fold change of ESG versus CG ).

\begin{tabular}{|c|c|c|c|c|}
\hline Gene ID & $P$ & Fold change & Symbol & Description \\
\hline 287443 & 0.0414 & 2.0120 & Acap1 & ArfGAP with coiled-coil, ankyrin repeat, and PH domains 1 \\
\hline 316628 & 0.0044 & 0.2748 & Asb1 & Ankyrin repeat and SOCS box-containing 1 (Asb1), mRNA \\
\hline 307970 & 0.0397 & 0.3289 & Atxn1l & $\begin{array}{l}\text { PREDICTED: similar to Ataxin-1 (Spinocerebellar ataxia } \\
\text { type } 1 \text { protein homolog) }\end{array}$ \\
\hline 304127 & 0.0266 & 0.4310 & Bach1 & $\begin{array}{l}\text { BTB and CNC homology 1, basic leucine zipper } \\
\text { transcription factor } 1\end{array}$ \\
\hline 94342 & 0.0368 & 0.4621 & Bat3 & HLA-B-associated transcript 3 , transcript variant 2 , \\
\hline 308588 & 0.0241 & 0.4679 & Car11 & Carbonic anhydrase-related XI protein \\
\hline 81780 & 0.0349 & 2.6298 & $\mathrm{Ccl} 5$ & Chemokine (C-C motif) ligand 5 \\
\hline 25405 & 0.0303 & 0.3845 & Ccng1 1 & Cyclin G1 \\
\hline 362217 & 0.0393 & 0.4273 & Cenpb & PREDICTED: centromere protein B \\
\hline 314004 & 0.0237 & 0.3330 & Cmpk2 & $\begin{array}{l}\text { Cytidine monophosphate (UMP-CMP) kinase } 2 \text {, } \\
\text { mitochondrial, nuclear gene encoding mitochondrial protein }\end{array}$ \\
\hline 24273 & 0.0401 & 0.4750 & Cryaa & Crystallin, alpha A \\
\hline 361729 & 0.0183 & 0.4488 & Cybasc3 & Cytochrome b, ascorbate dependent 3 \\
\hline 308942 & 0.0369 & 0.3530 & Dennd5a & DENN/MADD domain containing 5A \\
\hline 360583 & 0.0296 & 0.4192 & Dhrs11 & Dehydrogenase/reductase (SDR family) member 11 \\
\hline 362293 & 0.0203 & 0.4955 & Dnajb6 & DnaJ (Hsp40) homolog, subfamily B, member 6 \\
\hline 81655 & 0.0336 & 0.4654 & Dync1li2 & Dynein, cytoplasmic 1 light intermediate chain 2 \\
\hline 59117 & 0.0343 & 0.3116 & Eif2c2 & Eukaryotic translation initiation factor $2 \mathrm{C}, 2$ \\
\hline 497983 & 0.0476 & 0.4848 & Fam 117a & Family with sequence similarity 117 , member A \\
\hline 363083 & 0.0074 & 2.5213 & Fbxl22 & F-box and leucine-rich repeat protein 22 \\
\hline 29292 & 0.0293 & 0.4455 & Ftl & Ferritin, light polypeptide \\
\hline 54281 & 0.0281 & 0.3897 & Furin & Furin (paired basic amino acid cleaving enzyme) \\
\hline 25172 & 0.0185 & 0.3991 & Gata1 & GATA binding protein 1 \\
\hline 293267 & 0.0274 & 0.3516 & Hbe1 & Hemoglobin, epsilon 1 \\
\hline 94164 & 0.0175 & 0.4161 & $\operatorname{Hbg} 1$ & Hemoglobin, gamma A \\
\hline 498008 & 0.0335 & 2.2484 & Hexim 1 & Hexamethylene bis-acetamide inducible 1 \\
\hline 365895 & 0.0417 & 0.3894 & Hipk1 & Homeodomain interacting protein kinase 1 \\
\hline 288240 & 0.0022 & 0.3449 & Hlcs & $\begin{array}{l}\text { PREDICTED: holocarboxylase synthetase } \\
\text { (biotin-(proprionyl-Coenzyme A-carboxylase } \\
\text { (ATP-hydrolysing)) ligase) }\end{array}$ \\
\hline 293624 & 0.0080 & 0.3642 & Irf7 & Interferon regulatory factor 7 \\
\hline 290985 & 0.0079 & 0.4919 & Iscal & Iron-sulfur cluster assembly 1 homolog (S. cerevisiae) \\
\hline 298693 & 0.0462 & 0.3402 & Isg15 & ISG15 ubiquitin-like modifier \\
\hline 25118 & 0.0351 & 2.9262 & Itgal & Integrin, alpha 1 \\
\hline 300317 & 0.0493 & 0.4873 & Kctd17 & Potassium channel tetramerisation domain containing 17 \\
\hline 25110 & 0.0410 & 2.6060 & Klrd1 & Killer cell lectin-like receptor, subfamily D, member 1 \\
\hline
\end{tabular}


Table 9: Continued.

\begin{tabular}{|c|c|c|c|c|}
\hline Gene ID & $P$ & Fold change & Symbol & Description \\
\hline 245955 & 0.0120 & 0.4700 & Lgals3bp & Lectin, galactoside-binding, soluble, 3 binding protein \\
\hline 25476 & 0.0214 & 0.4406 & Lgals9 & Lectin, galactoside-binding, soluble, 9 \\
\hline 100365370 & 0.0172 & 0.4588 & LOC100365370 & $\begin{array}{l}\text { PREDICTED: nuclear LIM interactor-interacting factor } \\
\text { 2-like }\end{array}$ \\
\hline 498145 & 0.0213 & 0.3006 & LOC498145 & Similar to RIKEN cDNA 2810453106 \\
\hline 679596 & 0.0155 & 0.4814 & LOC679596 & $\begin{array}{l}\text { PREDICTED: similar to GABA(A) receptor-associated } \\
\text { protein like } 2\end{array}$ \\
\hline 684112 & 0.0121 & 0.4067 & LOC684112 & PREDICTED: similar to KIAA0999 protein \\
\hline 293156 & 0.0090 & 0.4140 & Lrtomt & $\begin{array}{l}\text { Leucine rich transmembrane and } 0 \text {-methyltransferase } \\
\text { domain containing }\end{array}$ \\
\hline 294241 & 0.0443 & 0.2072 & Ly6g6c & Lymphocyte antigen 6 complex, locus G6C \\
\hline 117558 & 0.0498 & 0.3267 & Mylk2 & Myosin light chain kinase 2 \\
\hline 85482 & 0.0360 & 0.4205 & Nbn & Nibrin \\
\hline 366998 & 0.0309 & 0.4486 & $\mathrm{Nfe} 2$ & Nuclear factor, erythroid derived 2 \\
\hline 59115 & 0.0355 & 0.3302 & Ninj2 & Ninjurin 2 \\
\hline 245980 & 0.0238 & 0.4878 & Nr2f6 & Nuclear receptor subfamily 2, group F, member 6 \\
\hline 287328 & 0.0292 & 0.4931 & Olr1439 & Olfactory receptor 1439 \\
\hline 287520 & 0.0498 & 0.4482 & Olr1516 & Olfactory receptor 1516 \\
\hline 366104 & 0.0175 & 0.4251 & Olr541 & Olfactory receptor 541 \\
\hline 246294 & 0.0120 & 0.3491 & Optn & Optineurin \\
\hline 362973 & 0.0467 & 0.4896 & Parvb & Parvin, beta \\
\hline 24649 & 0.0147 & 0.3899 & Pim1 & Pim-1 oncogene \\
\hline 64534 & 0.0423 & 2.1733 & Pim3 & Pim-3 oncogene \\
\hline 301173 & 0.0478 & 0.3759 & $\mathrm{Plcl} 2$ & Phospholipase C-like 2 \\
\hline 310674 & 0.0473 & 0.4134 & Plekho1 & Pleckstrin homology domain containing, family $\mathrm{O}$ member 1 \\
\hline 362873 & 0.0062 & 0.4404 & Plxnc1 & Plexin C1 \\
\hline 362248 & 0.0215 & 0.4759 & Procr & Protein $\mathrm{C}$ receptor, endothelial \\
\hline 309381 & 0.0286 & 2.2397 & Pyroxd2 & Pyridine nucleotide-disulphide oxidoreductase domain 2 \\
\hline 171452 & 0.0460 & 0.3652 & Rab3il1 & $\mathrm{RAB} 3 \mathrm{~A}$ interacting protein \\
\hline 56820 & 0.0334 & 0.1273 & Ramp3 & Receptor (G protein-coupled) activity modifying protein 3 \\
\hline 498659 & 0.0473 & 7.0377 & RatNP-3b & Defensin RatNP-3 precursor \\
\hline 296408 & 0.0259 & 0.4348 & RGD1311378 & Similar to RIKEN cDNA $2010011 \mathrm{I} 20$ \\
\hline 501644 & 0.0175 & 0.4259 & RGD1561055 & $\begin{array}{l}\text { PREDICTED: similar to Ferritin light chain } 2 \text { (Ferritin L } \\
\text { subunit 2) (Ferritin subunit LG) }\end{array}$ \\
\hline 65190 & 0.0454 & 0.3257 & Rsad2 & Radical S-adenosyl methionine domain containing 2 \\
\hline 24974 & 0.0165 & 0.4619 & RT1-A2 & RT1 class Ia, locus A2 (RT1-A2) \\
\hline
\end{tabular}


TABle 9: Continued.

\begin{tabular}{|c|c|c|c|c|}
\hline Gene ID & $P$ & Fold change & Symbol & Description \\
\hline 414779 & 0.0105 & 0.4766 & RT1-CE2 & RT1 class I, locus CE2 (RT1-CE2) \\
\hline 266758 & 0.0163 & 2.6183 & Sec11c & SEC11 homolog C (S. cerevisiae) \\
\hline 313057 & 0.0446 & 0.4886 & Serinc2 & Serine incorporator 2 \\
\hline 498546 & 0.0120 & 0.1863 & Serp2 & $\begin{array}{l}\text { Stress-associated endoplasmic reticulum protein family } \\
\text { member } 2\end{array}$ \\
\hline 360636 & 0.0484 & 0.4722 & Slc25a39 & Solute carrier family 25, member 39 (Slc25a39) \\
\hline 192208 & 0.0472 & 0.3469 & Slc38a5 & Solute carrier family 38, member 5 (Slc38a5) \\
\hline 300191 & 0.0457 & 0.4485 & Slc48a1 & Solute carrier family 48 (heme transporter), member 1 \\
\hline 64630 & 0.0330 & 0.4620 & Snap23 & Synaptosomal-associated protein 23 \\
\hline 314251 & 0.0353 & 0.4407 & Sptb & Spectrin, beta, erythrocytic \\
\hline 113894 & 0.0230 & 0.4367 & Sqstm 1 & Sequestosome 1 , transcript variant 1, mRNA \\
\hline 501146 & 0.0449 & 0.3749 & Stradb & STE20-related kinase adaptor beta \\
\hline 24851 & 0.0449 & 0.3944 & Tpm1 & Tropomyosin 1, alpha \\
\hline 303167 & 0.0390 & 0.3720 & Trim58 & Predicted: tripartite motif-containing 58 \\
\hline 362087 & 0.0450 & 0.3958 & Ubac1 & UBA domain containing 1 \\
\hline 295704 & 0.0234 & 0.3510 & Ube216 & Ubiquitin-conjugating enzyme E2L 6 \\
\hline 310633 & 0.0316 & 0.3751 & Ubqln 4 & Ubiquilin 4 \\
\hline 289229 & 0.0240 & 0.3468 & Vangl2 & Vang-like 2 \\
\hline 24874 & 0.0262 & 2.6865 & Vhl & Von Hippel-Lindau tumor suppressor \\
\hline 298765 & 0.0209 & 2.4995 & Zfp3612 & Zinc finger protein $36, \mathrm{C} 3 \mathrm{H}$ type-like 2 \\
\hline
\end{tabular}

genes, associated with overrepresentation of 36 biological processes and 34 canonical pathways indicating prenatal stress did not have to be experienced by the mother herself to influence offspring brain development. Among the GO annotations Itpr1 and Itpr2 appeared in almost all the affected pathways. In nonexcitable cells, the inositol 1,4,5-trisphosphate receptor (IP3R) is an intracellular $\mathrm{Ca}^{2 \mathrm{C}}$ channel, which plays a major role in $\mathrm{Ca}^{2 \mathrm{C}}$ signalling. Three isoforms of IP3R have been identified (IP3R-1, IP3R-2, and IP3R-3) and most cell types express different proportions of each isoform [31]. IP3Rs play major roles in agonistsinduced intracellular $\mathrm{Ca}^{2 \mathrm{C}}$ release and also in store operated $\mathrm{Ca}^{2 \mathrm{C}}$ entry, a process whereby the depletion of intracellular $\mathrm{Ca}^{2 \mathrm{C}}$ store causes the opening of $\mathrm{Ca}^{2 \mathrm{C}}$ channels in the plasma membrane [32]. The intracellular $\mathrm{Ca}^{2+}$ elevations induced by BDNF required a signaling pathway consistent with the activation of the Trk-IP3R cascade, which was also necessary for the activation of the membrane conductance IBDNF [33, 34]. Amaral and Pozzo-Miller [35] reported that Trk receptors, IP3Rs, full intracellular $\mathrm{Ca}^{2+}$ stores and $\mathrm{Ca}^{2+}$ influx are all required for BDNF-induced $\mathrm{Ca}^{2+}$ elevations and membrane currents. Opposing influences of mBDNF and proBDNF on long-term potentiation and long-term depression might contribute to the dichotomy of BDNF actions on behaviors mediated by the brain stress and reward systems $[36,37]$. Twelve KEGG pathways were annotated, including oocyte meiosis, vascular smooth muscle contraction, RIGI-like receptor signaling pathway, long-term potentiation, ubiquitin mediated proteolysis, and long-term depression, Titterness and Christie [38] prenatal ethanol and prenatal stress produce sex-specific alterations in synaptic plasticity in the adolescent hippocampus. Calpains, which belong to a family of at least 14 members of calcium-dependent cysteine proteases and are involved in apoptosis are implicated in a wide range of physiological functions including cell motility, differentiation, signal transduction, including cell survival pathways, cell cycle progression, regulation of gene expression, and long-term potentiation [39, 40]. Yang et al. [41] reported that prenatal stress (10 unpredictable, $1 \mathrm{~s}$, $0.8 \mathrm{~mA}$ foot shocks per day during gestational days 1319) impaired long-term potentiation (LTP) but facilitated long-term depression (LTD) in hippocampal CA1 region in slices of the prenatal stressed offspring ( 5 weeks old). Proteolysis by the ubiquitin-proteasome pathway has attained prominence as a new molecular mechanism which regulates varied important functions of the nervous system, including development of synaptic connections and synaptic plasticity 


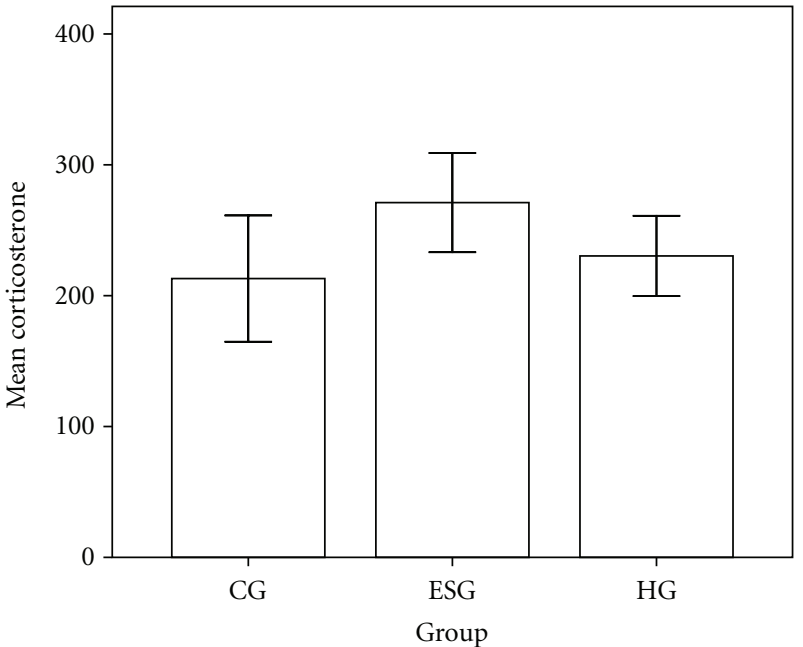

(a)

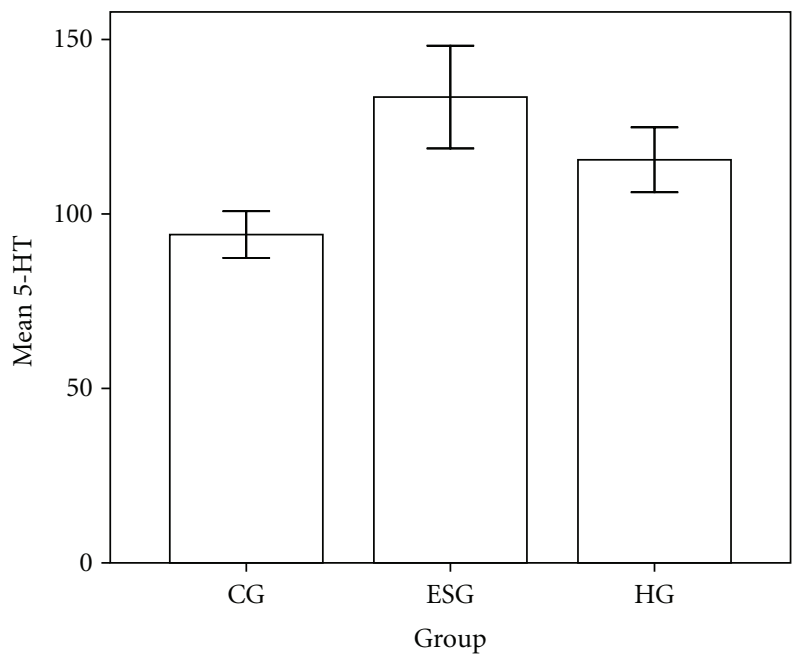

(c)

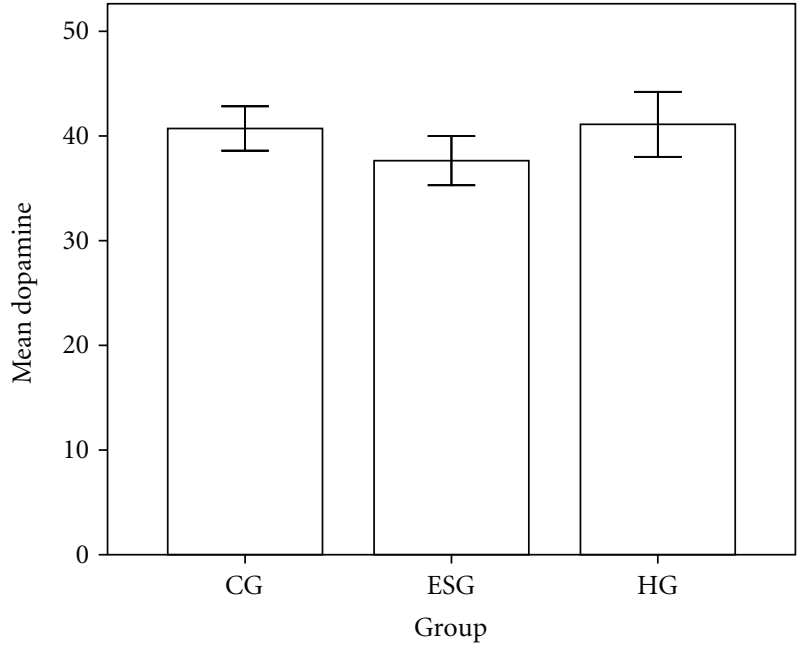

(b)

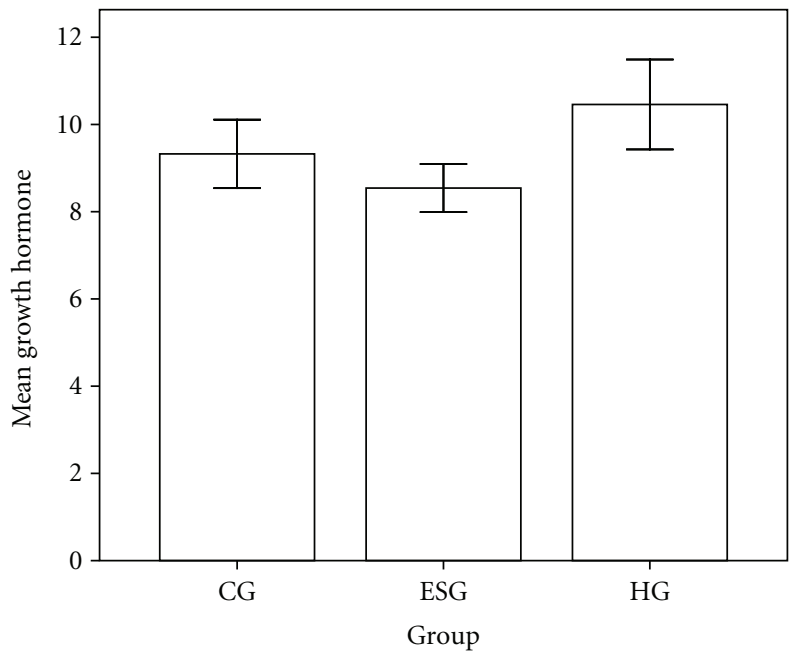

(d)

Figure 3: ELISA outcomes of corticosterone, dopamine, 5-HT, and growth hormone. (a) ANOVA test for the corticosterone showed $P=$ 0.027 in CG versus ESG, $P=0.491$ in CG versus HG, and $P=0.111$ in ESG versus HG. (b) ANOVA test for the dopamine showed $P=0.065$ in CG versus ESG, $P=0.805$ in CG versus HG, and $P=0.039$ in ESG versus HG. (c) ANOVA test for 5-HT showed $P=0.000$ in CG versus ESG, $P=0.004$ in CG versus HG, and $P=0.013$ in ESG versus HG. (d) ANOVA test for the growth hormone showed $P=0.135$ in CG versus ESG, $P=0.034$ in CG versus HG, and $P=0.001$ in ESG versus HG.

through control of axonal growth, axonal and dendritic pruning, and regulation of synaptic size and number [42].

We found 60 genes upregulated and 28 genes downregulated in HG versus ESG, from which five significant GO and five KEGG pathways were annotated, indicating diversified cellular biological process and signaling pathways. Interestingly, Socs 2 and Socs 4 of Socs (suppressors of cytokine signaling) family appeared in three of the KEGG pathways. SOCS family consists of eight structurally similar proteins (SOCS-1 to SOCS-7 and CIS), which have been implicated as potential inhibitors of tissue growth during both prenatal and postnatal life [43] and their actions clearly now extend to other intracellular pathways, they remain key negative regulators of cytokine and growth factor signaling [44]. Cytokinemediated JAK/STAT signaling, that is, Janus kinase/signal transducers and activators of transcription, controls a number of vital biologic responses, including immune function, cellular growth, differentiation, and hematopoiesis [45]. The SOCS Family-The SOCS proteins were identified as STAT target genes that directly antagonize STAT activation, resulting in a classic "feedback loop" [46]. PS in rats induced lifespan reduction of neurogenesis in the dentate gyrus and produced impairment in hippocampal-related spatial tasks through blocking the increase of learninginduced neurogenesis [47]. Previous research reported that male rats exposed to stress in utero are characterized by a decrease in hippocampal cell proliferation, and consequently neurogenesis, from adolescence to senescence [48]. PS has been reported to alter cytokine levels. CoussonsRead et al. [49] reported that stress-related neural immune 


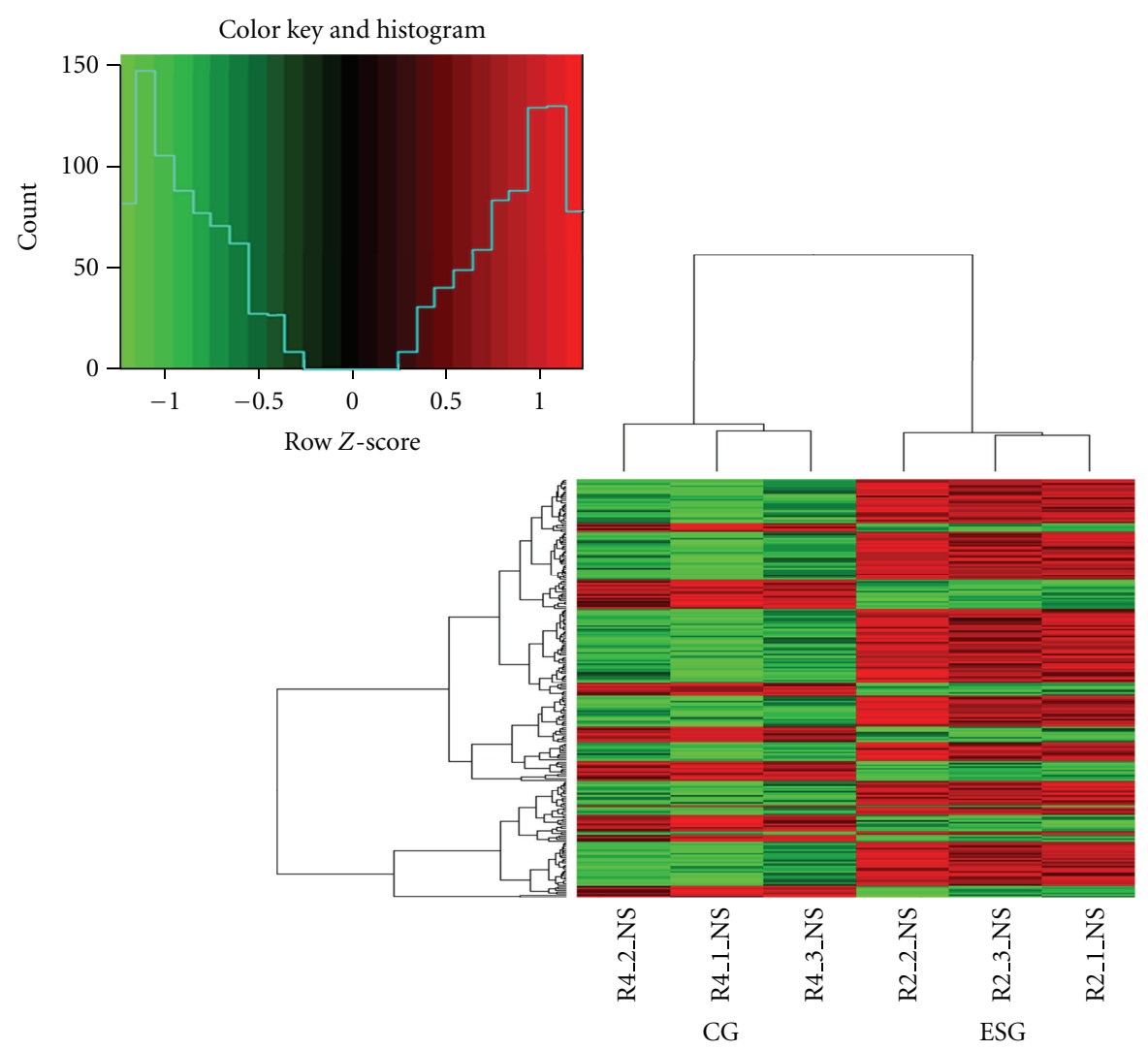

Figure 4: Heat map of the differently expressed genes. R2_1_NS, R2_2_NS, and R2_3_NS refer to ESG and R4_1_NS, R4_2_NS, R4_3_NS to CG.

interactions may contribute to pregnancy complications and poor outcome. Collier et al. [50] found that PS changed typical proinflammatory cytokines including tumor necrosis factor (TNF)- $\alpha$, and interleukin (IL)-6. As mentioned above, JKSQW recovered the dysfunction of kidney due to fear from earthquake, which could be supported by gene profile experiment outcome. In other words, cytokine conduction pathways, for example, JAK/STAT are involved in the prenatal kidney deficiency, and key molecules like Socs-2 and Socs-4 are the regulating targets of Chinese medicine treatment. The underlying mechanism that JKSQW improves development and behavior might attribute to the upregulation of Socs2 and Socs- 4 which suppress the pathway of JAK/STAT, resulting in reduction certain cytokines' secretion. diabetes is considered as Xiao-ke in Chinese medicine, whose major pattern is kidney deficiency. JKSQW plays an important role in the composition of prescriptions treating Diabetes in Chinese medicine [51]. Promisingly, our findings revealed insulin related pathways were involved in the outcome of herbal intervention in HG, supporting the hypnosis that JKSQW recovery the dysfunction of kidney.

Four genes (Irf7, Ninj2, Plxnc1, and Isca1) were validated with RT-PCR, showing a favorable match (75\%) between the gene expression profile chip and RT-PCR result. It is reported that all elements of IFN responses, whether the systemic production of IFN in innate immunity or the local action of IFN from plasmacytoid dendritic cells in adaptive immunity, are under the control of Irf7 [52]. Hannah et al. [53] reported that induction of pattern recognition receptors (PRRs; Tlr7 and Rig-I), expression of antiviral genes (Myd88, Visa, Jun, Irf7, Ifnbeta, Ifnar1, Jak2, Stat3, and $\mathrm{Mx} 2$ ), and production of $\mathrm{Mx}$ protein was elevated in the lungs of intact females compared with intact males. Ninjurin2 (Ninj2) is a transmembrane protein that mediates cell-to-cell and cell-to-extracellular matrix interactions during development, differentiation, and regeneration of the nervous system [54]. Recently, Ninj2 was reported to be a vascular susceptibility gene and associated with Alzheimer's disease risk [55].

In conclusion, together with our own recent data, the findings of this body of work demonstrate the earthquake as a prenatal stressor during the pregnancy could negatively retard the body and nervous system development, and Chinese herbal remedy could correct the retardation, which could attribute to neurohormones alteration and altered gene expression profile. The gene pathways involved have been tied to signaling pathway, long-term potentiation, ubiquitin mediated proteolysis, and long-term depression relating to disruptions from prenatal stress; Jak-STAT signaling pathway could play a key role in improving the function of JKSQW. This study demonstrates that negatively prenatal experiences have the ability to significantly retard 


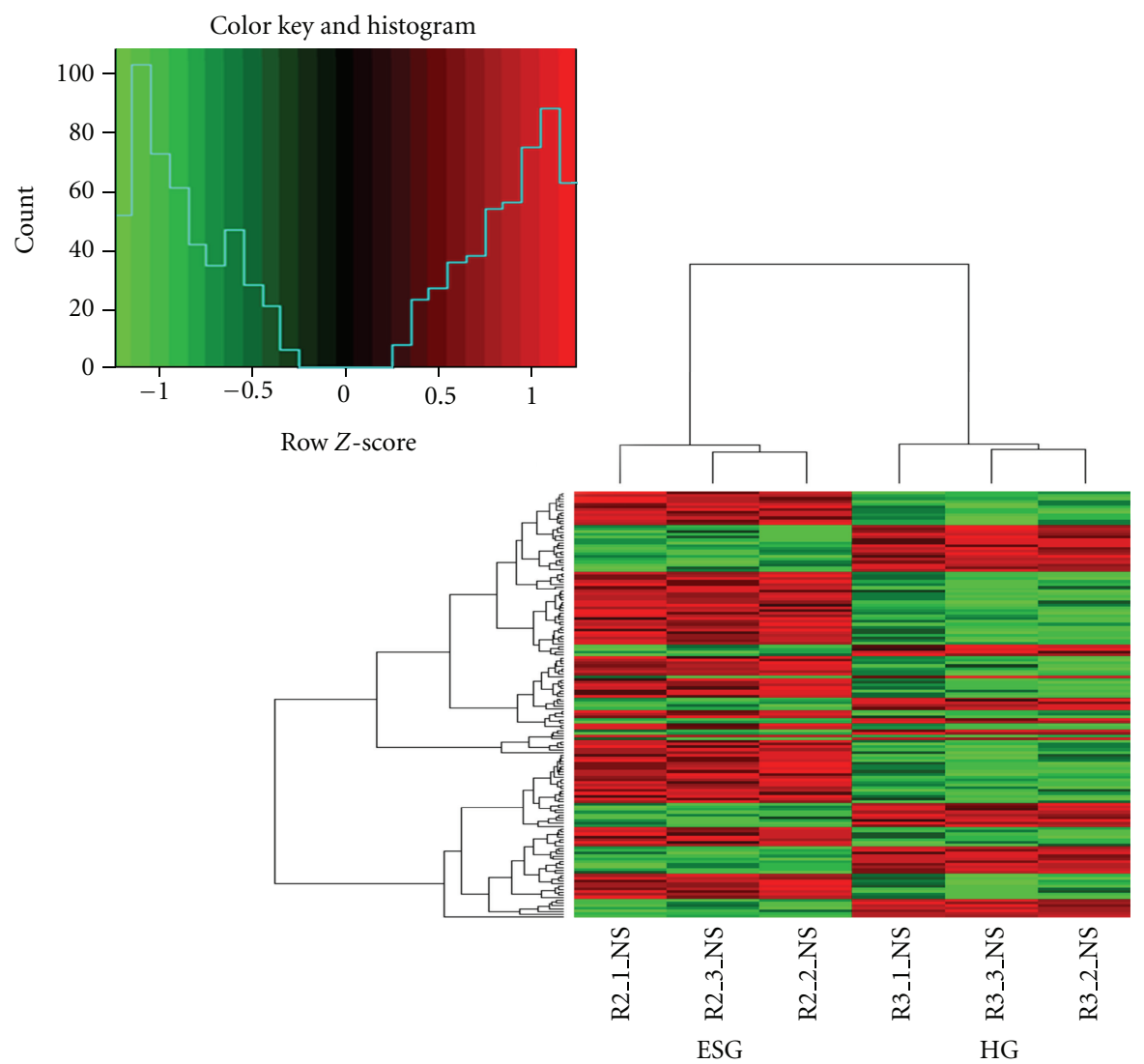

FIGURE 5: Heat map of the differently expressed genes. R2_1_NS, R2_2_NS, and R2_3_NS refer to ESG and R3_1_NS, R3_2_NS, R3_3_NS to HG.

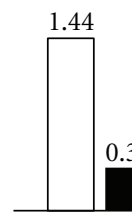

Irf7

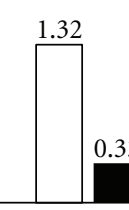

Ninj2

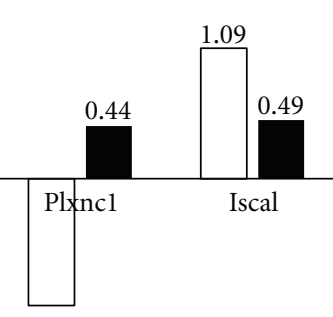

$-1.06$

$\Delta \Delta$ Ct of RT-PCR

$\mathrm{FC}$ of gene expression

(a) Relationship of rt-PCR and gene expression (ESG versus CG)

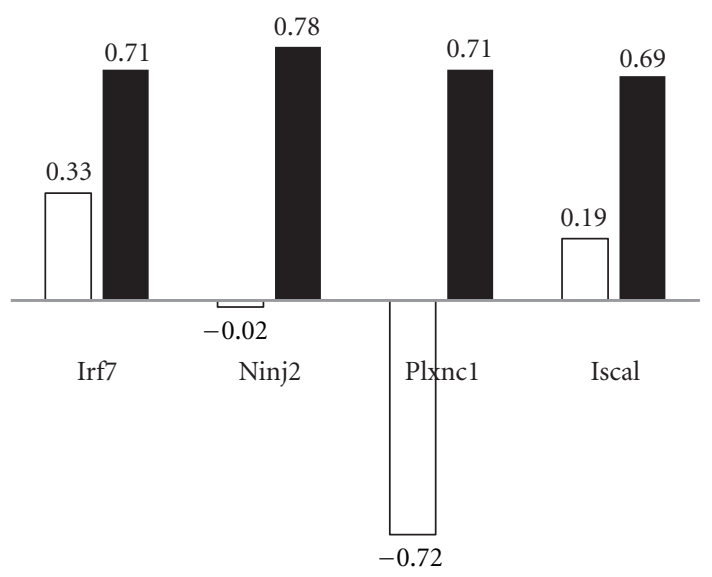

$\triangle \Delta$ Ct of RT-PCR

FC of gene expression

(b) Relationship of rt-PCR and gene expression (HG versus CG (no change))

FIGURE 6: RT-PCR validation of the selected four genes from gene expression profile chips, that is, Irf7, Ninj2, and Plxnc1, and Isca1. $\Delta \Delta \mathrm{Ct}<0$ indicates the target genes were hyperexpressed in ESG/HG comparing with CG while $\Delta \Delta \mathrm{Ct}>0$ indicates the target genes were hypoexpressed in ESG/HG comparing with CG. FC > 2 indicates the target genes were hyperexpressed in ESG/HG comparing with CG while $\mathrm{FC}<0.5$ indicates the target genes were hypoexpressed in ESG/HG comparing with CG. 
offspring developmental and immunity trajectories, which can be corrected by Chinese herbal remedy.

\section{Conflict of Interests}

The authors declare no conflict of interests.

\section{Acknowledgments}

This study was under the support of National Science Funds of China with the Grant no. 81072719. The authors thank Sheri L. Johnson, Ph.D. and Zeyiad Elias, Ph.D. USA for English editing.

\section{References}

[1] J. Simpson and J. P. Kelly, "The impact of environmental enrichment in laboratory rats-Behavioural and neurochemical aspects," Behavioural Brain Research, vol. 222, no. 1, pp. 246 264, 2011.

[2] K. O'Donnell, T. G. O'Connor, and V. Glover, "Prenatal stress and neurodevelopment of the child: focus on the HPA axis and role of the placenta," Developmental Neuroscience, vol. 31, no. 4, pp. 285-292, 2009.

[3] S. Morley-Fletcher, M. Rea, S. Maccari, and G. Laviola, "Environmental enrichment during adolescence reverses the effects of prenatal stress on play behaviour and HPA axis reactivity in rats," European Journal of Neuroscience, vol. 18, no. 12, pp. 3367-3374, 2003.

[4] E. J. H. Mulder, P. G. Robles De Medina, A. C. Huizink, B. R. H. Van Den Bergh, J. K. Buitelaar, and G. H. A. Visser, "Prenatal maternal stress: effects on pregnancy and the (unborn) child," Early Human Development, vol. 70, no. 1-2, pp. 3-14, 2002.

[5] S. Maccari, M. Darnaudery, S. Morley-Fletcher, A. R. Zuena, C. Cinque, and O. Van Reeth, "Prenatal stress and long-term consequences: implications of glucocorticoid hormones," Neuroscience and Biobehavioral Reviews, vol. 27, no. 1-2, pp. 119-127, 2003.

[6] S. Maccari and S. Morley-Fletcher, "Effects of prenatal restraint stress on the hypothalamus-pituitary-adrenal axis and related behavioural and neurobiological alterations," Psychoneuroendocrinology, vol. 32, pp. S10-S15, 2007.

[7] J. Lesage, F. Del-Favero, M. Leonhardt et al., "Prenatal stress induces intrauterine growth restriction and programmes glucose intolerance and feeding behaviour disturbances in the aged rat," Journal of Endocrinology, vol. 181, no. 2, pp. 291296, 2004.

[8] S. King, R. G. Barr, A. Brunet, J. F. Saucier, M. Meaney et al., "The ice storm: an opportunity to study the effects of prenatal stress on the baby and the mother," Santé Mentale au Québec, vol. 25, no. 1, pp. 163-185, 2000.

[9] C. E. Tan, H. J. Li, X. G. Zhang et al., "The impact of the Wenchuan earthquake on birth outcomes," PLoS ONE, vol. 4, no. 12, Article ID e8200, 2009.

[10] C. Oyarzo, P. Bertoglia, R. Avendaño et al., "Adverse perinatal outcomes after the February 27th 2010 Chilean earthquake," Journal of Maternal-Fetal and Neonatal Medicine, vol. 25, no. 10, pp. 1868-1873, 2012.

[11] R. Yaka, S. Salomon, H. Matzner, and M. Weinstock, "Effect of varied gestational stress on acquisition of spatial memory, hippocampal LTP and synaptic proteins in juvenile male rats," Behavioural Brain Research, vol. 179, no. 1, pp. 126-132, 2007.
[12] P. Leung, M. Cheung, and V. Tsui, "Help-seeking behaviors among Chinese Americans with depressive symptoms," Social Work, vol. 57, no. 1, pp. 61-71, 2012.

[13] A. Kolasani, H. Xu, and M. Millikan, "Determination and comparison of mineral elements in traditional chinese herbal formulae at different decoction times used to improve kidney function-chemometric approach," The African Journal of Traditional, Complementary and Alternative Medicines, vol. 8, supplement 5, pp. 191-197, 2011.

[14] X. G. Zhang, Y. Q. Yang, S. T. Li et al., "Further study on the thought of terror impairing kidney by simulating the earthquake experiment," J Sichuan Trandit Chin Med, vol. 26, no. 12, pp. 27-28, 2008.

[15] O. Kofman, "The role of prenatal stress in the etiology of developmental behavioural disorders," Neuroscience and Biobehavioral Reviews, vol. 26, no. 4, pp. 457-470, 2002.

[16] E. C. Cottrell and J. R. Seckl, "Prenatal stress, glucocorticoids and the programming of adult disease," Frontiers in Behavioral Neuroscience, vol. 3, article 19, 2009.

[17] M. Weinstock, "The long-term behavioural consequences of prenatal stress," Neuroscience and Biobehavioral Reviews, vol. 32, no. 6, pp. 1073-1086, 2008.

[18] Y. Kotozaki and R. Kawashima, "Effects of the Higashi-Nihon earthquake: posttraumatic stress, psychological changes, and corticosterone levels of survivors," PLoS ONE, vol. 7, no. 4, Article ID e34612, 2012.

[19] R. S. Goland, S. Jozak, W. B. Warren, I. M. Conwell, R. I. Stark, and P. J. Tropper, "Elevated levels of umbilical cord plasma corticotropin-releasing hormone in growth-retarded fetuses," Journal of Clinical Endocrinology and Metabolism, vol. 77, no. 5, pp. 1174-1179, 1993.

[20] Y. Huang, H. Xu, H. Li, H. Yang, Y. Chen, and X. Shi, "Pregestational stress reduces the ratio of 5-HIAA to 5-HT and the expression of 5-HT1A receptor and serotonin transporter in the brain of foetal rat," BMC Neuroscience, vol. 13, article 22, 2012.

[21] S. Spinelli, S. Chefer, R. E. Carson et al., "Effects of earlylife stress on serotonin ${ }_{1 A}$ receptors in juvenile rhesus monkeys measured by positron emission tomography," Biological Psychiatry, vol. 67, no. 12, pp. 1146-1153, 2010.

[22] M. K. Brown and Y. Luo, "Bilobalide modulates serotonincontrolled behaviors in the nematode Caenorhabditis elegans," BMC Neuroscience, vol. 10, article 62, 2009.

[23] S. Davidson, D. Prokonov, M. Taler et al., "Effect of exposure to selective serotonin reuptake inhibitors. In Utero on fetal growth: potential role for the IGF-I and HPA axes," Pediatric Research, vol. 65, no. 2, pp. 236-241, 2009.

[24] I. C. G. Weaver, A. C. D'Alessio, S. E. Brown et al., "The transcription factor nerve growth factor-inducible protein a mediates epigenetic programming: altering epigenetic marks by immediate-early genes," Journal of Neuroscience, vol. 27, no. 7, pp. 1756-1768, 2007.

[25] E. Carboni, V. G. Barros, M. Ibba, A. Silvagni, C. Mura, and M. C. Antonelli, "Prenatal restraint stress: an in vivo microdialysis study on catecholamine release in the rat prefrontal cortex," Neuroscience, vol. 168, no. 1, pp. 156-166, 2010.

[26] Z. Y. Shen and D. P. Cai, "Study on the regulative rule of reinforcing shen principle on sexual precocity and senescence at the molecular level," Zhongguo Zhong Xi Yi Jie He Za Zhi, vol. 25, no. 6, pp. 549-551, 2005.

[27] R. H. Mak, W. W. Cheung, and C. T. Roberts Jr., “The growth hormone-insulin-like growth factor-I axis in chronic kidney disease," Growth Hormone and IGF Research, vol. 18, no. 1, pp. 17-25, 2008. 
[28] Y. H. Yang and Z. Li, "Gene chip study of cerebral genome of effect of jinkui shenqi pill in mice model with kidney-yang asthenia induced by excessive physical and sexual activity," Liaoning Journal of Traditional Chinese Medicine, vol. 35, no. 5, pp. 733-779, 2008.

[29] W. Gao, J. Paterson, M. Abbott, S. Carter, and L. Iusitini, "Maternal mental health and child behaviour problems at 2 years: findings from the Pacific Islands Families Study," Australian and New Zealand Journal of Psychiatry, vol. 41, no. 11, pp. 885-895, 2007.

[30] C. J. Ewell Foster, J. Garber, and J. A. Durlak, "Current and past maternal depression, maternal interaction behaviors, and children's externalizing and internalizing symptoms," Journal of Abnormal Child Psychology, vol. 36, no. 4, pp. 527-537, 2008.

[31] R. Mychasiuk, N. Schmold, S. Ilnytskyy, O. Kovalchuk, B. Kolb, and R. Gibb, "Prenatal bystander stress alters brain, behavior, and the epigenome of developing rat offspring," Developmental Neuroscience, vol. 33, no. 2, pp. 159-169, 2011.

[32] G. Arguin, Y. Regimbald-Dumas, M. O. Fregeau, A. Z. Caron, and G. Guillemette, "Protein kinase $\mathrm{C}$ phosphorylates the inositol 1,4,5-trisphosphate receptor type 2 and decreases the mobilization of $\mathrm{Ca}^{2+}$ in pancreatoma AR4-2J cells," Journal of Endocrinology, vol. 192, no. 3, pp. 659-668, 2007.

[33] J. T. Smyth, W. I. DeHaven, B. F. Jones et al., "Emerging perspectives in store-operated $\mathrm{Ca}^{2+}$ entry: roles of Orai, Stim and TRP," Biochimica et Biophysica Acta, vol. 1763, no. 11, pp. 1147-1160, 2006.

[34] H. Nakata and S. Nakamura, "Brain-derived neurotrophic factor regulates AMPA receptor trafficking to post-synaptic densities via IP3R and TRPC calcium signaling," FEBS Letters, vol. 581, no. 10, pp. 2047-2054, 2007.

[35] M. D. Amaral and L. Pozzo-Miller, "TRPC3 channels are necessary for brain-derived neurotrophic factor to activate a nonselective cationic current and to induce dendritic spine formation," Journal of Neuroscience, vol. 27, no. 19, pp. 51795189, 2007.

[36] M. D. Amaral and L. Pozzo-Miller, "BDNF induces calcium elevations associated with IBDNF, a nonselective cationic current mediated by TRPC channels," Journal of Neurophysiology, vol. 98, no. 4, pp. 2476-2482, 2007.

[37] K. Martinowich, H. Manji, and B. Lu, "New insights into BDNF function in depression and anxiety," Nature Neuroscience, vol. 10, no. 9, pp. 1089-1093, 2007.

[38] A. K. Titterness and B. R. Christie, "Prenatal ethanol exposure enhances NMDAR-dependent long-term potentiation in the adolescent female dentate gyrus," Hippocampus, vol. 22, no. 1, pp. 69-81, 2012.

[39] S. J. Franco and A. Huttenlocher, "Regulating cell migration: calpains make the cut," Journal of Cell Science, vol. 118, no. 17, pp. 3829-3838, 2005.

[40] D. E. Goll, V. F. Thompson, H. Li, W. Wei, and J. Cong, "The calpain system," Physiological Reviews, vol. 83, no. 3, pp. 731801, 2003.

[41] J. Yang, H. Han, J. Cao, L. Li, and L. Xu, "Prenatal stress modifies hippocampal synaptic plasticity and spatial learning in young rat offspring," Hippocampus, vol. 16, no. 5, pp. 431436, 2006.

[42] A. N. Hegde and S. C. Upadhya, "The ubiquitin-proteasome pathway in health and disease of the nervous system," Trends in Neurosciences, vol. 30, no. 11, pp. 587-595, 2007.

[43] S. Gentili, J. S. Schwartz, M. J. Waters, and I. C. McMillen, "Prolactin and the expression of suppressor of cytokine signaling-3 in the sheep adrenal gland before birth," American Journal of Physiology, vol. 291, no. 5, pp. R1399-R1405, 2006.
[44] B. A. Croker, H. Kiu, and S. E. Nicholson, "SOCS regulation of the JAK/STAT signalling pathway," Seminars in Cell and Developmental Biology, vol. 19, no. 4, pp. 414-422, 2008.

[45] R. N. Cooney, "Suppressors of cytokine signaling (SOCS): inhibitors of the JAK/STAT pathway," Shock, vol. 17, no. 2, pp. 83-90, 2002.

[46] W. S. Alexander and D. J. Hilton, "The role of Suppressors of Cytokine Signaling (SOCS) proteins in regulation of the immune response," Annual Review of Immunology, vol. 22, pp. 503-529, 2004.

[47] V. Lemaire, M. Koehl, M. Le Moal, and D. N. Abrous, "Prenatal stress produces learning deficits associated with an inhibition of neurogenesis in the hippocampus," Proceedings of the National Academy of Sciences of the United States of America, vol. 97, no. 20, pp. 11032-11037, 2000.

[48] M. Koehl, V. Lemaire, M. Le Moal, and D. N. Abrous, "Age-dependent effect of prenatal stress on hippocampal cell proliferation in female rats," European Journal of Neuroscience, vol. 29, no. 3, pp. 635-640, 2009.

[49] M. E. Coussons-Read, M. L. Okun, M. P. Schmitt, and S. Giese, "Prenatal stress alters cytokine levels in a manner that may endanger human pregnancy," Psychosomatic Medicine, vol. 67, no. 4, pp. 625-631, 2005.

[50] C. T. Collier, P. N. Williams, J. A. Carroll, T. H. Welsh, and J. C. Laurenz, "Effect of maternal restraint stress during gestation on temporal lipopolysaccharide-induced neuroendocrine and immune responses of progeny," Domestic Animal Endocrinology, vol. 40, no. 1, pp. 40-50, 2011.

[51] H. Zhang, C. E. Tan, H. Z. Wang, S. B. Xue, and M. Q. Wang, "Study on the history of traditional Chinese medicine to treat diabetes," European Journal of Integrative Medicine, vol. 2, no. 1, pp. 41-46, 2010.

[52] K. Honda, H. Yanai, H. Negishi et al., "IRF-7 is the master regulator of type-I interferon-dependent immune responses," Nature, vol. 434, no. 7034, pp. 772-777, 2005.

[53] M. F. Hannah, V. B. Bajic, and S. L. Klein, "Sex differences in the recognition of and innate antiviral responses to Seoul virus in Norway rats," Brain, Behavior, and Immunity, vol. 22, no. 4, pp. 503-516, 2008.

[54] T. Araki and J. Milbrandt, "Ninjurin2, a novel homophilic adhesion molecule, is expressed in mature sensory and enteric neurons and promotes neurite outgrowth," Journal of Neuroscience, vol. 20, no. 1, pp. 187-195, 2000.

[55] K. P. Lin, S. Y. Chen, L. C. Lai et al., "Genetic polymorphisms of a novel vascular susceptibility gene, ninjurin2 (NINJ2), are associated with a decreased risk of Alzheimer's disease," PLoS ONE, vol. 6, no. 6, Article ID e20573, 2011. 


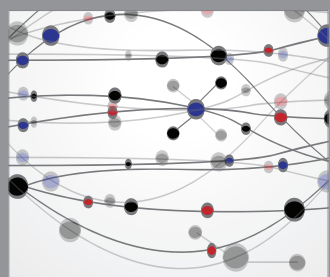

The Scientific World Journal
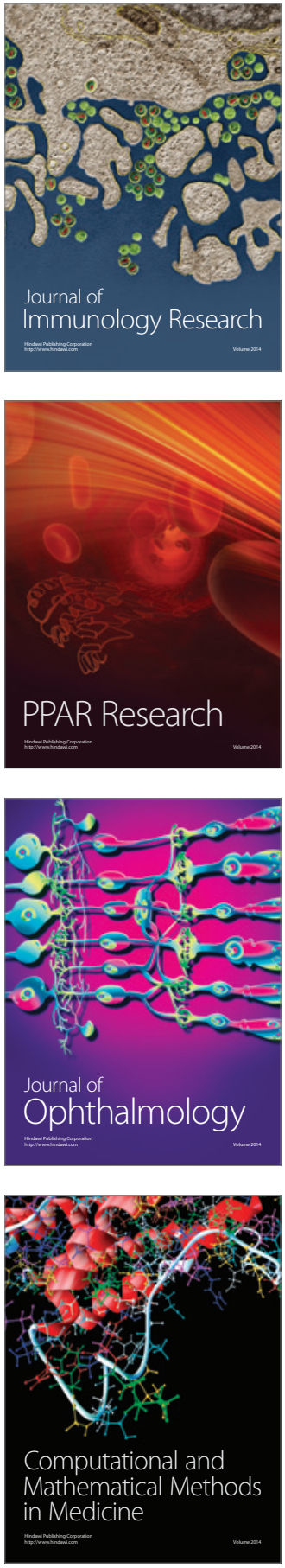

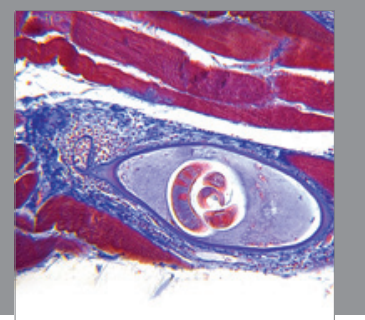

Gastroenterology

Research and Practice
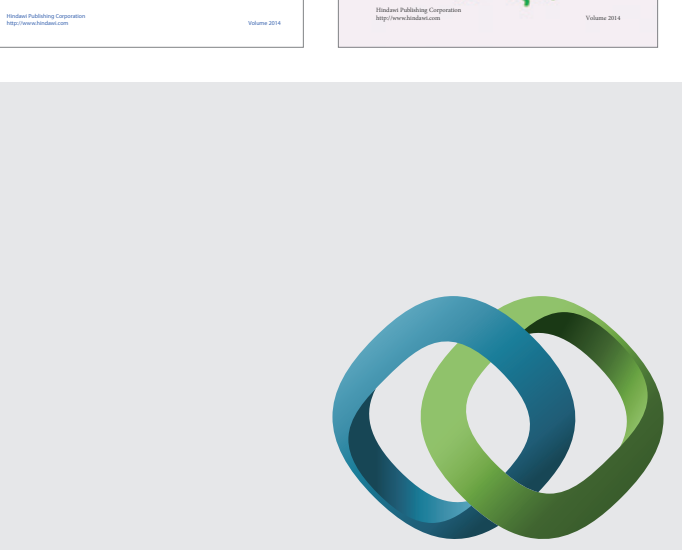

\section{Hindawi}

Submit your manuscripts at

http://www.hindawi.com
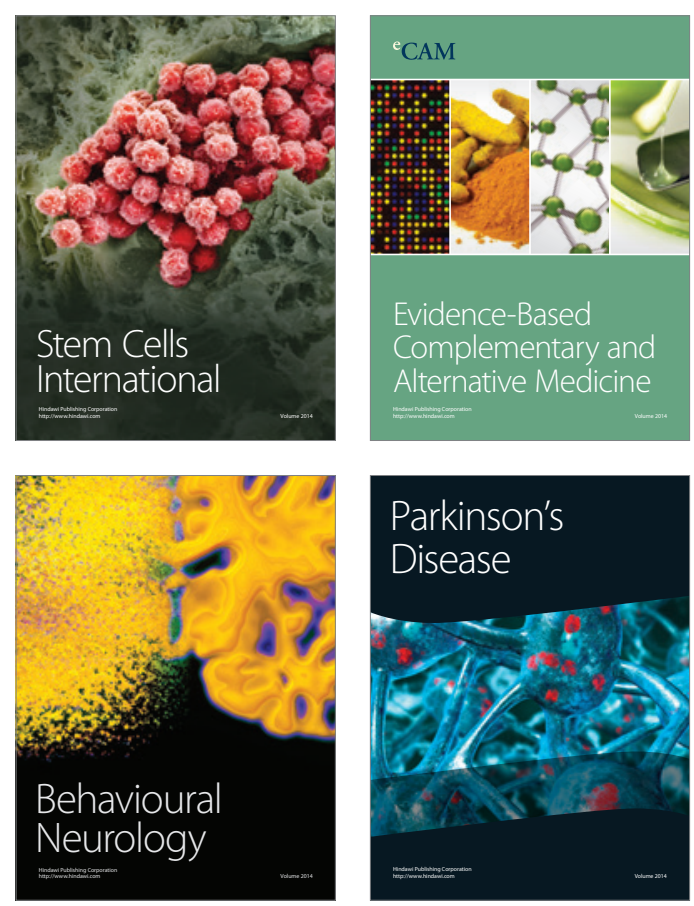

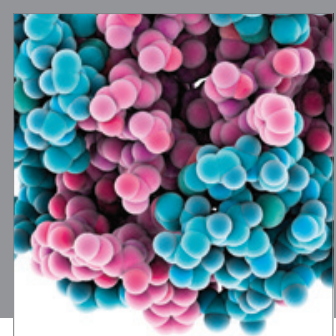

Journal of
Diabetes Research

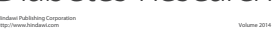

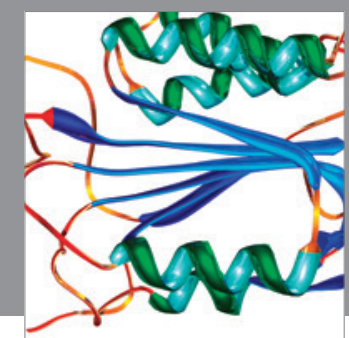

Disease Markers
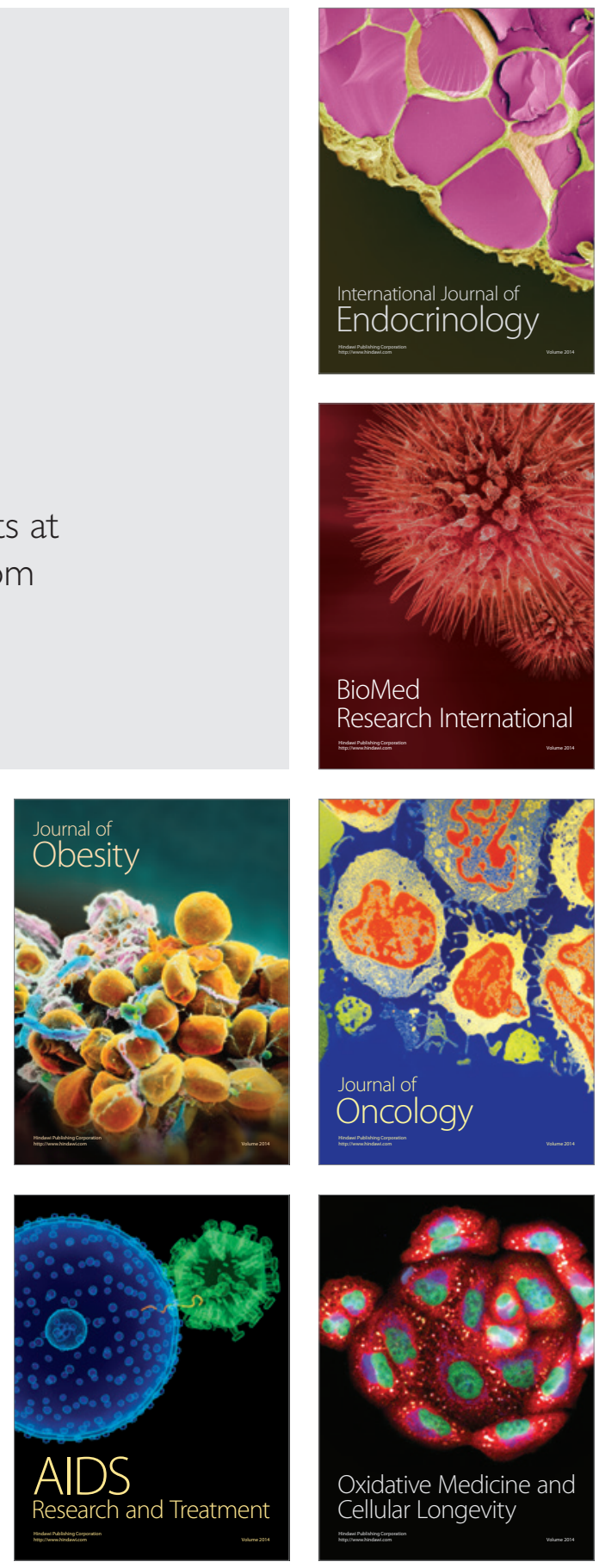